\title{
Ueber den Einfluss \\ des hohen Blutdruckes auf die Neubildung der Cerebrospinalflüssigkeit.
}

\author{
Von
}

\author{
Hofrath Dr. A. Spima in Prag.
}

Die Frage nach der Bildung des Liquor cerebrospinalis hat durch eine Reihe von Versuchen, welche ich vor Kurzem veröffentlicht habe, eigentlich schon ihre Beantwortung gefunden ${ }^{1}$ ). Ich theilte damals mit, dass der Liquor von den hyperämischen Gehirngefässen bei hohem Blutdrucke transsudirt wird.

Die Versuche, welche dieser Behauptung zur Stütze gereichen sollten, waren in Kürze die folgenden:

Wird einem curaresirten Hunde Nebennierensaft intravenös injicirt, so wird das entblösste Gehirn hyperämisch, etwas grösser und feuchter, oder - und diese Erscheinung habe ich besonders hervorgehoben - es treten, während das Gehirn in Folge der Hyperämie anschwillt, aus demselben Liquortröpfchen zur Oberfäche empor. In einem bedeutenderen Grade macht sich diese Erscheinung geltend, wenn man einen Lähmungsprolaps, und noch auffallender wird dieselbe, wenn man einen Druckprolaps des Gehirns hervorruft.

Den ersteren erzielte ich, wenn ich das obere Halsmark bei uneröffnetem Schädel zerdrückt und tamponirt und hierauf nach Entblössung des Gehirns den Nebennierenextract eingespritzt habe. Die Gehirnhyperämie nimmt jetzt an Intensität zu, das Gehirn schwillt bis zum deutlichen Prolaps an und die Tröpfchenbildung an dessen Oberfläche wird stärker. Im Kopf- und oberen Halsmarke, sagte ich, befindet sich ein vasoconstrictorisches Centrum, von welchem Bahnen nach aufwärts zu den Gefässen des Gebirns ziehen. Wird dieses Centrum zerstört und der Blutdruck durch die Injection des Nebennierenextractes erhöht, so werden die gelähmten Hirngefässe

1) Experimentelle Untersuchungen über die Bildung des Liquor cerebrospinalis. Pflüger's Archiv Bd. 76. 1899. 
von dem Blutdrucke ausgedehnt ${ }^{1}$ ) und mit Blut überfüllt und zwar an stärksten in dem entblössten Gehirnantheile, weil hier der Widerstand am geringsten ist. Aus diesen Gründen wird das Gehirn roth, prolabirt und bedeckt sich unter den Augen des Beobachters mit zahlreichen Tropfen von Liquor. Da hierbei die Lähmung der Gehirngefässe den wesentlichen Ausschlag gibt, habe ich diese Art von Prolaps als "Lähmungsprolaps" bezeichnet.

Trennt man aber das obere Kopf- öder Halsmark nach der Entblössung einer Gehirnpartie ohne Tamponade durch, indem man nur durch Verschluss der äusseren Wunde dafür sorgt, dass keine Blutung nach Aussen erfolgt, so werden die Hirngefässe gleichfalls gelähmt, der Blutdruck steigt in Folge der mit der Durchschneidung verbundenen mechanischen Reizung der Oblongata an, das entblösste Gehirn wird roth und voluminöser. Da aber noch der Druck von Seite der Extravasates hinzutritt, wird das Gehirn durch die Trepanöffnung gewaltsam nach Aussen gedrängt. Unter den eben geschilderten Versuchsbedingungen ist die Prolaps- und Liquorbildung am grössten. Bei dem Umstande, dass der Hauptgrund der beschriebenen Erscheinung in dem Drucke des aus den durchschnittenen Gefässen austretenden Blutes gegeben ist, nannte ich diesen Hirnvorfall der Kürze des Ausdruckes wegen "Druckprolaps".

Ist hingegen das vasoconstrictorische Centrum unverletzt, so wird das Gehirn nach der Extractinjection zwar auch hyperämisch, aber in einem geringeren Grade, wie aus der unbeträchtlichen Volumsvergrösserung der entblössten Gehirnpartie und der geringeren Liquorbildung ersichtlich ist.

Es gibt aber, wie ich in der citirten Abhandlung mitgetheilt habe, noch eine dritte Methode, einen Gehirnprolaps hervorzurufen.

Ich habe die Aorta an ihrer Wurzel comprimirt und dann nach 6 Minuten die Compression beseitigt. Das Gehirn, das in Folge der Anämie sich verkleinerte und erblasste, wurde nach der Wiedereinführung des Kreislaufes hyperämisch und vergrösserte, wie dies schon $\mathrm{Kussmaul}$ und T enner ${ }^{2}$ ) beobachtet haben, sein Volumen derart, dass dasselbe etwas grösser wurde als zu Beginn des Versuches. Wahrseheinlicherweise handelt es sich hier um die schon

1) $\mathrm{Ob}$ die Gefässdilatation nur auf mechanische Weise erfolgt, liess ich dahingestellt. So viel ist sicher, dass man mit der mechanischen Erklärung der Gehirnhyperamie derzeit sein Auslangen findet.

2) Moleschott's Untersuchungen Bd. 3 . 
wiederholt beobachtete "Superdilatation" der Gefässe in Folge der Anämisirung des Gehirns. Aber zur Entwicklung eines grösseren Prolapses kommt es nicht, dieselbe tritt erst ein, wenn unmittelbar nach der Herstellung des Kreislaufes der Nebennierenextract injicirt wird. Auch dieser Prolaps wird oft von vermehrter Liquorbildung begleitet.

Eine brauchbare Erklärung für diese Art von Prolapsbildung glaubte ich in der Annähme gefunden zu haben ${ }^{1}$ ), dass durch die Blutleere des Gehirns die vasoconstrictorischen Apparate ausser Function gesetzt werden und dass demgemäss die Blutgefässe desselben dem in Folge der Extrartinjection herandrängenden Blute keinen Widerstand zu leisten vermögen.

Eine directe Ernährungsstörung der Gehirngefässe durch die Anämie konnte nicht als der entscheidende Grund in Betracht gezogen werden, da ich erfahren habe, dass der Prolaps grösser wirł, wenn der Versuch mit der Ligirung der Aorta nach vorangegangener Zerstörung der Oblongata ausgeführt wird ${ }^{2}$ ). Wenn man alle diese Erfahrungen zu Rathe zieht, so kann nur der Vorstellung Raum gegeben werden, dass auch der nach Ligirung der Aorta und Einwirkung des Extractes erscheinende Prolaps eigentlich auch ein Lähmungsprolaps ist.

Es wurde des Weiteren von mir dargethan, dass der auf der Oberfläche des entblössten Gehirnes erseheinende Liquor aus den Blutgefässen des Gehirnes selbst stammt, und dass die Wege, welche derselbe hierbei einschlägt, aller Wahrscheinlichkeit zufolge die extraoder intraadventiellen Lymphräume der Gehirngefässe vorstellen.

Die Beobachtung, dass der Liquor auf der Oberfläche des entblössten Gehirns zu Tage tritt, hat ihre Bestätigung in einer von R. $\mathrm{Fuchs}^{3}$ ) ausgeführten Untersuchung gefunden. Es geschah dies aber in einer Weise, welche geeignet ist, bei dem Leser die unrichtige Vorstellung wachzurufen, als ob Fuchs die Erscheinung schon vor mir gesehen hätte, denn die Worte, dass ich das Hervorperlen des Liquors $a \mathrm{uch}$ beobacitet habe, lassen meine Priorität als zweifelhaft erseheinen.

1) Moleschott's Untersuchungen $\mathrm{Bd} .3$.

2) Näheres hierüber 1. c. S. 211 und 212.

3) Zur Regulirung der Blutcirculation im Gehirn. Sitzungsber. d. deutschen naturw.-medic. Vereins für Böhmen. "Lotos" $1899 \mathrm{Nr}$. 3. 
Bevor ich in der Mittheilung meiner neueren Untersuchungen weiter schreite, will ich die eben genannte Publication einer kritischen Auseinandersetzung unterziehen.

Zwei Gründe sind es, die mich dazu veranlassen. Erstens enthält die Abhandlung eine Reihe von Beobachtungen, welche meine Angaben bestätigen - auf eine dieser Angaben habe ich schon hingewiesen -, und andererseits gelangt der Autor der Publication zu Schlussfolgerungen, welche mit meinen Erfahrungen in Widerspruch gerathen. Ich hege aber hierbei keineswegs die Absicht, nur zu polemisiren, denn ich werde gelegentlich Beobachtungen einfügen, welche meine früher gemachten Erfahrungen zu ergänzen geeignet sind. Es soll vorerst der erste Punkt seine Erledigung finden.

Dr. Fuchs bestätigt, dass die durch Injectionen von Nebennierenextracten hervorgerufene Gehirnhyperämie bei unverletztem Kopf- und Halsmarke keinen Prolaps bedingt, dass während der Paquelinisirung des oberen Halsmarkes gleichfalls kein Prolaps zur Entwicklung gelangt. Dr. Fuchs bestätigt ferner, dass die von Knoll gegen meine Angaben gerichtete Behauptung, dass die von mir beschriebenen Prolapse nur durch das Extravasat bewirkte Druckprolapse seien, unrichtig ist; denn Fuchs berichtet, dass die Prolapsbildung auch ohne Extravasat zu Stande kommt. Endlich hat Fuchs conform meinen Angaben auch Druckprolapse mit Berstung der Gehirnventrikel hervorgerufen.

Mit diesen Beobachtungen erscheint eine Reihe von Versuchsergebnissen, mit denen ich meine Theorie bewähren wollte, genügend beglaubigt.

Dr. Fuchs bringt aber die angeführten Erfahrungen in andere Beziehungen zu einander, als dies von mir geschehen ist.

So soll die von mir als Lähmungsprolaps bezeichnete Erscheinung - nach hoher Markdurchtrennung - nicht durch Lähmung der Vasoconstrictoren, sondern durch die Cerebrospinalflüssigkeit hervorgerufen werden. Die hierbei dem Liquor zugetheilte Aufgabe besteht in einem Vorgange, den Dr. Fuchs als "Selbsttamponade" näher bezeichnet. Dieser Vorgang soll wie folgt ablaufen:

Wird der Blutdruck bei einem Thiere mit trepanirtem Schädel und durchschnittener Dura erhöht, so quillt das Gehirn auf, und aus der Schädelöffnung fliesst der von dem sich vergrössernden Gehirne verdrängte Liquor nach Aussen. Unter continuirlichem Abflusse des Liquors erreicht dann das quellende Gehirn die Ränder der Tre- 
panationsöffnung und verstopft die letztere wie ein Tampon. Nun ist dem Liquor der Weg für den Abfluss verlegt und „in dem Maasse, als neue Cerebrospinalflüssigkeit zufolge des gesteigerten Blutdruckes sich bildet", wird die weitere Ausdehnung des Gehirnes durch den Druck von Seite des Liquors verhindert, und das Gehirn kann sich nur noch im Bereiche der Schädelöffnung ausdehnen, es kommt zum Prolaps.

Die durch die Selbsttamponade bedingte Anhäufung des Liquors ist demnach Fuchs zufolge die Hauptursache des Prolapses, die Blutung ist zum Zustandekommen des Gehirnvorfalles nicht nothwendig. Wohl kann aber, wenn das Halsmark verletzt worden ist, das Extravasat im Vereine mit dem Liquor die Prolapsbildung beschleunigen. Alles, was die Selbsttamponade fördert, fördert nach den Angaben von Fuchs auch den Prolaps. Es wird demzufolge eine kleinere und an tiefer gelegenen Stellen des Schädels angebrachte Oeffnung das Vorfallen des Gebirns begünstigen. Liegt hingegen die Trepanationsöffnung hoch, so wird sie von dem quellenden Gehirne nicht erreicht, der Liquor kann sich nicht ansammeln und der Prolaps bleibt aus.

Ich habe dieser Darstellung Folgendes entgegenzustellen:

Vor Allem ist zu bemerken, dass sich Fuchs bei der Deutung der geschilderten Vorgänge nicht treu bleibt. Auf Seite 14 des Separatabdruckes erscheint die Selbsttamponade als die Ursache und die Anhäufung des Liquors als die Folge angegeben, aber auf Seite 16 steht der volle Gegensatz davon, "es wird durch den behinderten Liquorabfluss die Selbsttamponade erreicht". Was ist also das Richtige? Schon dieser Widerspruch könnte mich jeder weiteren Kritik entheben.

Die oben mitgetheilte Erklärung über die Prolapsbildung jst ferner mit meinen Beobachtungen unvereinbar. Der von Fuchs behauptete Ausfluss von Liquor nach der Erhöhung des Blutdruckes und die Anhäufung desselben nach der Selbsttamponade gehört nicht zu den regelmässigen Ereignissen bei der Prolapsbildung. Man kann zwar eine Ansammlung des Liquors oft beobachten, wenn man das Rückenmark nahe dem distalen Ende der Halswirbelsäule - etwa am dritten oder vierten Halswirbel - durchschneidet und tamponirt. Der Grund hiervon ist einleuchtend. In dem drei bis vier Wirbel langen Rückgradscanale kann es wohl zur Anhäufung von Liquor kommen. Wird aber das Kopfmark in der Gegend der Membrana 
obturatoria posterior durchtrennt und tamponirt und dann der Schädel trepanirt und das Gehirn entblösst, dann fliesst, was an Liquor vorbanden ist, nahezu Alles $a b$, und trotzdem pflegen gerade bei dieser Präparationsweise die Prolapse deutlich zu sein. Auch wenn nach erfolgter Prolapsbildung mittelst einer zwischen Hirn und Dura eingeführten Sonde nach dem Liquor gefahndet wird, ist eine Ansammlung desselben nicht zu constatiren, vorausgesetzt, dass der Abfluss der Cerebrospinalflüssigkeit nach Spaltung" der Dura ein ausgiebiger war.

Es ist des Weiteren - offenbar durch ungenigende Lectüre Dr. Fuchs unbekannt geblieben, dass ich in der von ihm kritisirten Publication ${ }^{1}$ ) Versuche mitgetheilt habe, in denen die Hälfte oder das ganze Schädeldach mit der Dura abgetragen wurde und trotzdem der Prolaps eingetreten ist. Es ist nicht denkbar, dass unter solchen Versuchsbedingungen der Hirnvorfall durch Selbsttamponade und Ansammlung von Liquor entstanden ist.

Auch jene Versuche, in welchen der Prolaps durch Anämisirung und darauffolgende Extractinjection hervorgerufen wird, machen es klar, dass die von Fuchs behauptete Liquoransammlung mit dem Prolapse in keinem ursächlichen Zusammenhange steht. Bei diesen Versuchen kann nämlich der Prolaps wiederholt an demselben Thiere erzeugt werden. Der Liquor müsste sich hier wiederholt anstauen und bei der Trepanationsöffnung abfliessen, und doch ist davon, das Hervorsickern von Liquortropfen auf der Oberfläche des Prolapses ausgenommen, nichts zu sehen.

Die Lage der Trepanationsöffnung ist gleichfalls für die Entstehung des Hirnvorfalls nur von einer untergeordneten Bedeutung, denn das Gehirn prolabirt bei den mannigfaltigsten Variationen in der Lage derselben. Ja, es unterliegt keinen Schwierigkeiten, an demselben Schädel zwei und mehr Vorfälle an Contrastellen gleichzeitig hervorzurufen.

Diese ganze Kette von Beobachtungen lehrt, dass sich die Angaben Dr. Fuchs' auf dem Boden eines Irrthums bewegen. Fuchs hat offenbar nur einige wenige Versuche ausgeführt und auf Grundlage dieses unzureichenden Materials seine Schlüsse gefasst.

Der von Dr. Fuchs gegebenen Erklärung fällt aber noch ein anderer Fehler zur Last.

1) Experimentelier Beitrag zur Kenntniss der Hyperamie des Gehirns. Wiener medic. Blätter 1898 Nr. 16 und 17. 
Fuchs behauptet, dass die Selbsttamponade für den Prolaps eine unumgängliche Bedingung ist. Controlversuche an Thieren mit intactem Rückenmarke hätten ihn aber eines Anderen belehrt. Trepanirt man den Schädel eines kräftigen, schwach curarisirten Hundes, so wird man, wenn der Blutdruck nicht gesunken ist, des Oefteren gewahr, dass das entblösste Gehirn schon vor der Extractinjection die Trepanationsöfnung tamponirt. Wird nun der Extract injicirt, so erhebt sich der Blutdruck oft bis über $300 \mathrm{~mm} \mathrm{Hg}$, das Gehirn wird roth und schwillt etwas an. Wiewohl bei dieser Versuchsanordnung alle Bedingungen, welche Fuchs als nothwendig zur Entstehung des Gehirnvorfalles bezeichnet, gegeben sind, tritt derselbe dennoch nicht ein.

Es fällt mir jedoch nicht ein, zu bestreiten, dass die Weite der Trepanationsöffnung, ihre Lage und der Liquor den Prolaps absolut nicht beeinflussen könnten. Aber darauf muss ich bestehen, dass diese Beeinflussung eine unwesentliche ist.

Es kann aber andererseits gezeigt werden, dass auch beim Fehlen aller jener Bedingungen, welche nach $\mathrm{Fuchs}$ für die Entstehung der Prolapses unabweislich nothwendig sind, sich trotzdem der Vorfall des Gehirns entwickelt. Der Versuch, durch welchen das Gesagte dargelegt werden soll, beruht auf der von mir gemachten Erfahrung, dass nach grossen Gaben von Curare (8-10 ccm einer $2 \%$ igen Lösung intravenös injicirt) das Gehirn auffallend collabirt. Der Versuch selbst wurde in folgender Weise ausgeführt:

Einem schwach curaresirten Hunde. wurde das Kopfmark in der Gegend der Membrana obturatoria mittelst eines Wattestückes zerdrückt und tamponirt und hierauf $10 \mathrm{ccm}$ Curare in Pausen von je einer Minute injicirt. Wird nun der Schädel an seinem höchsten Punkte trepanirt und die Dura durchtrennt, so findet man das Gehirn verkleinert und zwischen Schädel und Dura einen deutlichen liquorfreien Zwischenraum.

Es wird nun der eben bereitete 5\% ige Nebennierenextract (in physiologischer Kochsalzlösung) intravenös injicirt. Der Blutdruck erhebt sich, das Gehirn wird roth, schwillt an, jener Zwischenraum wird nun vom Gehirne ausgefüllt und dieses selbst prolabirt. Der Prolaps ist nicht gross, aber doch deutlich. Fuchs zufolge hätte sich hier kein Prolaps entwickeln, es hätte nicht zur Selbsttamponade kommen sollen, da hier alle Bedingungen zur Prolabirung des Gehirns gefehlt haben. Trotzdem kam es zu der 
Selbsttamponade, und nicht allein das, das Gehirn tamponirte durch seine Schwellung sowohl die Trepanationsöffnung als auch den ganzen Schädelraum.

Dr. Fuchs strengt weiterhin den Beweis an, dass der Prolaps auch bei Intactsein des von mir behaupteten vasoconstrictorischen Centrums sich entwickeln könne. Er zerstörte mit dem Glüheisen das Halsmark im oberen Theile des vierten Segmentes und tamponirte die Wunde. Nach Injection des Extractes trat ein Prolaps ein. Da in diesem Versuche, deducirt Fuchs, "das angebliche Centrum erhalten geblieben" und der Prolaps trotz alledem auftrat, so kann nicht die Lähmung der Gehirnvasoconstrictoren die Ursache des Gehirnvorfalls abgeben.

Diese Schlussfolgerung beruht auf einer falschen Prämisse, und zwar auf der Prämisse, dass das vasoconstrictorische Centrum vor dem vierten Halssegmente endigt. Diesem Irrthume wäre der Autor entgangen, wenn er meine Publication, bevor er an ihre Kritik herangetreten ist, besser gelesen hätte. Ich habe in derselben auf Seite 10 des Separatabdruckes ${ }^{1}$ ) angegeben: "Nahezu dasselbe Resultat - Hyperämie des Gehirns ohne Prolapsbildung - ergibt eine Reihe von Versuchen, in denen das Rückenmark in verschiedenen Höhen bis zum unteren Rande des vierten Halswirbels durchschnitten worden ist." Schon damals habe ich es des Weiteren als fraglich bezeichnet, ob mittelst der Methode der Prolapserzeugung die Grenzen jenes Centrums und seiner Bahnen strenge bestimmbar sind. Denn die Prolapse werden, je weiter man vom dritten Halswirbel nach abwärts das Mark durchschneidet, kleiner, und es hängt dann sehr viel vou dem subjectiven Ermessen ab, ob die geschwellte Hirnpartie noch als Prolaps aufzufassen ist oder nicht.

In den Angaben von Dr. Fuchs ist aber noch eine andere Ungenauigkeit enthalten. Ich messe in meiner Publication nach Wirbeln, Dr. Fuchs aber nach Segmenten. Wie gross die Differenz zwischen beiden Maassen ist, vermag ich im gegenwärtigen Augenblicke nicht zu bestimmen, und Dr. Fuchs, an dem die Reihe gewesen wäre, sein Maass mit dem von mir gewählten zu vergleichen, ist dieser Verschiedenheit des Maasses schlechterdings nicht gewahr

1) Experimentelle Untersuchungen über den Einfluss von Rückenmarksdurchtrennungen auf den Kreislauf des Gehirns. Wiener klin. Wochenschrift $1897 \mathrm{Nr} .48$. 
geworden. Es kann aber, da die Segmente zumeist höher liegen als die entsprechenden Wirbel, mit grosser Wahrscheinlichkeit gefolgert werden, dass die Zerstörung des Rückenmarkes im vierten Segmente noch in den unteren Bereich des dritten Wirbels gefallen ist. Dr. Fuchs war somit nicht berechtigt, diesen Versuch gegen mich anzuspielen, deun es ist nicht ausgeschlossen, dass derselbe meine Behauptung von der Existenz jenes Centrums gegen den Willen des Dr. Fuchs bestätigt. Ja ich kann auf Grundlage von neueren Versuchen bebaupten, dass dies nicht nur nicht ausgeschlossen, sondern direct erwiesen werden kann. Ich erzielte bei einem Hunde, dem ich das Rückenmark in der Höbe des vierten Halswirbels durchtrennt habe, nach der Extractinjection einen ganz deutlichen Hirnvorfall. Aber hei der Wiederholung des Versuches an einem anderen Hunde war der Prolaps schon bedeutend kleiner. Es kann demgemäss nicht bezweifelt werden, dass das Centrum und seine aus ihm tretenden Bahnen noch in der Höhe des vierten Wirbels, wenn auch nicht immer, nachweisbar sind. Ob dieser Wirbel die thatsächliche untere Grenze vorstellt, kann ich aber trotzdem noch immer nicht behaupten.

Es wird fernerhin von Dr. Fuchs behauptet, dass es ibm nicht gelungen ist, bei Kaninchen nach der Durchschneidung des oberen Halsmarkes und Extractinjection einen Prolaps hervorzurufen. Das ist auch leicht zu begreifen, denn $\mathrm{Fu}$ chs erwähnt selbst gelegentlich der Discussion dieser Versuche, dass der Blutdruck nicht genügt bat, um das Gehirn bis zur Selbsttamponade zu vergrössern. Ein hoher Blutdruck ist aber nach meinen Angaben für die Prolapsbildung eine conditio sine qua non. Im Uebrigen habe ich, wie aus meinen $A b-$ handlungen hervorgeht, an Kaninchen sehr wenig experimentirt. Publicirt habe ich nur einen Versuch ${ }^{1}$ ) und diesen habe ich unter Bedingungen ausgefuhrt, welche von denen, unter welchen Fuchs experimentirte, in vielen Stücken abweichen.

Ueber die übrigen von $\mathrm{Fuchs}$ angestellten Versuche ist nichts von Belang zu berichten. Die Protokolle sind so knapp mitgetbeilt; Momente, auf welche Alles ankommt, wie die Höhe des Blutdruckes nach der Extractinjection, werden nicht erwähnt. Um nur ein Beispiel anzuführen, löffelt $\mathbf{F}$ u chs das erste Segment des Halsmarkes ohne besondere Blutung aus. Wie er aber dabei vorgegangen, dass

1) Wiener klin. Wochenschr. 1897 Nr. 48. 
der hohe Blutdruck in Folge der mechanischen Reizung an der angefuhrten Stelle keine heftige Blutung veranlasst hat, wird dem Leser vorenthalten. Eine Volumsvermehrung des Gehirns bis zu der Lamina vitrea fasst Fuchs als Prolaps auf. Nun kann man sich ohne Mühe überzeugen, dass das Gehirn sehr oft de norma der Vitrea anliegt oder die Trepanationsöffnung ausfiult. Von einem Prolaps kann doch nur dann gesprochen werden, wenn das Gehirn über die Tabula externa deutlich hinausragt. Am zwanglosesten lassen sich die Resultate nach der Auslöffelung dadurch erklären, dass Fuchs entweder an einem körperlich heruntergekommenen Hunde experimentirt hat oder dass das Thier während des Versuches geschädigt worden ist. Eine verjässliche Kritik ist bei der Lückenhaftigkeit des Protokolles nicht möglich. Ich kann mit Bestimmtheit behaupten, dass geschwächte Hunde, welche hungernd und abgezehrt dem Institute geliefert worden sind, weder zu Versuchen über den Lähmungsprolaps, ja nicht einmal zur Hervorrufung eines Druckprolapses geeignet waren. Der Grund hiervon ist oft darin gelegen, dass die Extractinjection bei ihnen nur eine unbedeutende oder kurzwährende Steigerung des Blutdruckes bewirkt. In anderen Fällen scheint die geringe Blutmenge das veranlassende Moment zu sein. Ich berufe mich diesbezüglich auf die Erfahrung, dass bei Hunden nach grösserer Entnahme von Blut, wenn auch der Blutdruck nach der Extractinjection gestiegen ist, kein Lähmungsprolaps hervorzurufen ist.

Aber auch wenn die Versuchsthiere gut gepflegt erscheinen, gibt es $a b$ und zu Ausnahmen von der von mir aufgestellten Regel. So habe ich bei einem curaresirten Hunde, dem der Schädel trepanirt und die Dura durchtrennt worden war, beobachtet, dass das Hirn ohne jeden weiteren Versuchseingriff plötzlich prolabirt ist. Der Grund dieser Erscheinung ist mir nicht bekannt, denn ich habe dieselbe unter mindestens 80 Fällen nur einmal gesehen. Möglicherweise lag hier ein plötzlicher Nachlass in der Function des vasoconstrictorischen Centrums vor.

Ein anderes Mal trat trotz der Durchschneidung des obersten Halsmarkes und der Extractinjection kein Prolaps auf. Da ich aber gleichzeitig den Blutdruck verzeichnet habe, bin ich der Bedenken, die sich an diesen Versuch knüpfen konnten, los geworden. Gleich nach der Injection stieg nämlich der Blutdruck auf $310 \mathrm{~mm} \mathrm{Hg}$ an, fiel aber nach etwa drei Secunden wieder auf $108 \mathrm{~mm}$ herab. Das 
Gehirn wurde zwar roth und etwas grösser, aber zum Prolaps kam es nicht, weil der Blutdruck inzwischen gesunken ist. Auch dieser Erscheinung bin ich nur einmal begegnet. Es ist somit klar, dass unter den Mitteln, welche für mein Beweisverfahren in Betracbt kommen, die Beobachtung des Blutdruckes in erster Reihe steht, und darum soll sie bei den Prolapsversuchen zur Regel gemacht werden. Was für Waffen würde Dr. Fuchs aus diesen Versuchen gegen mich geschmiedet haben, wenn er zufällig auf diese Ausnahmsfälle gerathen wäre!

Noch auf andere Ausnahmsfälle bin ich gestossen. Ich habe Hämorrhagien aus stärkeren Gefässen in der Regel nur an prolabirten Gehirnen gesehen, vorausgesetzt, dass bei der Entblössung des Gehirns keine Gefässe desselben verletzt worden sind.

In jüngster Zeit habe ich aber drei Mal Blutungen ohne Prolaps nach der Extractinjection bei intactem Rückenmarke beobachtet. Die Widerstandsfähigkeit der Gefässe ist offenbar nicht bei allen Thieren die gleiche. In der uberwiegenden Mehrzahl der Fälle treten aber diese Blutungen nicht ein.

Ich habe gegen die Untersuchungen Dr. Fuchs' noch Folgendes auzuführen. Die experimentelle Forschung über den Kreislauf des Gehirns verfügt über eine Methode, mittelst welcher bei unverletztem Cranium unter Berücksichtigung des allgemeinen Blutdruckes und der aus dem Gehirne fliessenden Blutmenge auf die Innervation der Blutgefässe geschlossen werden kann. Die Methode stanmt von G. Gärtner und Jul. Wagner her ${ }^{1}$ ). Auch nach der technischen Richtung hin haben die genannten Autoren ihre Methode ausgebildet und gezeigt, welche von den Hirnvenen und unter welchen Cautelen dieselbe benutzt werden kann.

Fuchs griff die Angaben von Gärtner und Wagner und gleichzeitig auch mich an, weil auch ich nach der Methode der angeführten Autoren gearbeitet habe. Der Haupteinwand, den Fuchs erhob, lautete, dass es nicht angehe, aus der Menge des aus einem Organe ausfliessenden Blutes auf den Zustand des Gefässlumens zu schliessen, und stellte die Sache so dar, als ob Gärtner und Wagner nur den Ausfluss und nicht auch den Blutdruck beobachtet hätten.

Ein anderer von Dr. Fuchs erhobener Einwand war gegen den anatomischen Theil der von Gärtner und Wagner angegebenen

1) Wiener medic. Wochenschr. 1887. 
Methode gerichtet. Es könnte, meint Dr. Fuchs, der zahlreichen Anastomosen wegen das Blut aus den äusseren Schädelgebieten in die von Gärtner und Wagner benützte Vene, trotzdem dieselbe durch Ligirung aller nebensächlichen Seitenzweige isolirt wird, sich ergiessen.

Gärtner und Wagner haben ihre Angaben Fuchs gegenüber vertheidigt ${ }^{1}$ ) und fassen ihr Urtheil wie folgt zusammen: "Da uns also Dr. Fuchs vorwirft, dass wir einen Umstand nicht berücksichtigt haben (die Messung des Blutdruckes) dem wir thatsächlich und zwar in eingebender Weise Rechnung getragen haben, so müssen wir, um nicht zu noch ungünstigeren Schlüssen gedrängt zu sein, annehmen, dass Herr Dr. Fuchs unseren Aufsatz gar nicht gelesen hat." - "Wir bekennen aufrichtig, dass wir die Annahme des Herrn Dr. Fuchs (dass sich die Ausflussmenge vermehren könne ohne Aenderung des Gefässlumens, wenn in einem Bezirke der äusseren Schädelgefässe der Widerstand anwächst) für grundfalseh halten. Es ist merkwürdig, dass Herr Dr. Fuchs es wagt, solche Thesen aufzustellen, obne es der Mühe werth zu finden, sie durch ein Experiment zu prüfen."

Ich schliesse mich dem vollen Inhalte dieser Kritik an, sehe mich aber noch genöthigt, in Bezug auf die Anwendung der von Fuchs gerügten Methode Folgendes zu bemerken:

Ich habe nach Injection des Nebennierenextractes ein Ansteigen des Aortendruckes mit gleichzeitiger Vermehrung des ausfliessenden Blutes auch aus der von Gärtner und Wagner empfohlenen Hirnvene beobachtet und durfte mir demgemäss keinen Rückschluss auf die Grösse des Gefässquerschnittes erlauben; denn, ist die Ausflussmenge vermehrt und der Blutdruck in der Aorta erböht, so folgt nicht daraus, dass die Hirngefässe weiter geworden sind, denn es könnte der Blutstrom an Schnelligkeit gewonnen haben. Aber ich habe am entblössten Gehirn direct beobachtet, dass sich die Gefässe nach der Extractinjection erweitern, und daraus auf den Zustand derselben bei intactem Cranium geschlossen. Diese Schlussfolgerung wäre nicht gestattet, stände die alte Lehre von Al. Monro und Abercrombie zu Recht, der zufolge der unzusammenärückbare Inhalt des Craniums eine Aenderung der Strombreite des Gehirnblutes nicht zulasse. Da aber diese Lehre längst abgethan ist, da ferner der Blutdruck nach der Extractinjection eine exorbitante Höhe erreicht

1) Wiener klin. Wochenschr. 1899 Nr. 26. 
und das Blut in grossen Mengen aus dem Gehirne fliesst, wäre es ein Wunder, wenn die Gefässe dabei genau dieselbe Weite beibehalten sollten wie vor der Injection. Nicht ich, sondern Derjenige, der die Unwandelbarkeit des Gefässquerschnittes unter den eben angegebenen Bedingungen behauptet, hat den Beweis für dieselbe anzutreten.

Ich habe übrigens zum Ueberflusse auch die Erweiterung der Gehirngefässe bei intactem Schädel nach der alten Methode des Einsetzens eines Glasfensters dargethan ${ }^{1}$ ).

In Betreff der Art und Weise, wie ich dabei vorgegangen, möchte ich hier meiner Zusage gemäss nachträglich Folgendes bemerken: Nach Trepanirung des Schädels und nachdem die Blutung aus der Diploë sistirt hat, wird die Umgebung der Trepanationsöfnung sorgfältig getrocknet und durch Aufgiessen von heissem Wachse zu einer ebenen Fläche planirt. Sobald das Wachs erstarrt ist, legt man, während auf die trepanirte Stelle aus einem grösseren Gefässe vorgewärmte physiologische Kochsalzlösung geschüttet wird, über die Oeffnung eine kleine Glastafel ${ }^{2}$ ), trocknet rasch ab und bestreicht die letztere an ihren Rändern abermals mit heissem Wachse. Durch das Fenster hindurch sieht man nun das Gehirn nach der Injection roth und, wenn der Blutdruck abgefallen ist, wieder blässer werden. Entfernt man die Glasdecke zu einer Zeit, in welcher die Röthe des Gehirns in Folge der Injection deutlich geworden ist, so wird das Gehirn noch röther, in ähnlicher Weise, wie ich dies beobachtet habe, wenu man das Gehirn, nachdem der Blutdruck künstlich erhöht worden war, rasch trepanirt ${ }^{3}$ ).

In Bezug auf die Einwände, welche Fuchs gegen die Präparationsweise jener Vene, aus welcher der Abfluss des venösen Blutes beobachtet wird, geltend gemacht hat, habe ich Folgendes anzuführen:

Abgesehen davon, dass schon Gärtner und Wagner-Fuchs entgegnet haben, dass das von ihm behauptete Einströmen von Blut aus den oberflächlichen Schädelgebieten in jene Vene auf unerwiesenen Vermuthungen beruht, will ich $\mathrm{Fu} \mathrm{ch}$ s für den Augenblick concediren, dass jene unerwiesene Vermuthung erwiesen wäre und trotzdem zeigen, dass sein Einwand ohne Werth ist.

Es ist experimentell sichergestellt - Fuchs ist dies aber un-

1) $\mathbf{P}$ flüger's Archiv Bd. 76.

2) Ich benutzte Glastafeln ron $18 \mathrm{~mm}$ im Quadrat.

3) Wiener klin. Wochenschr. 1897 Nr. 48 S. 26 des Separatabdruckes. 
bekannt geblieben - , dass sich unter der Einwirkung des Nebellnierenextractes die kleinen Gefässe in den meisten Gebieten - das Gehirn, die Lunge und vielleicht die Retina ausgenommen - zusammenziehen, und in Bezug auf den Kopf kann man, wie Velich ${ }^{1}$ ) angegeben, die Beobachtung machen, dass die Zunge, das Zahnfleisch, die Mund-, die Nasenschleimhant und die Ohren blass werden und es so lange bleiben, als der Blutdruck hoch bleibt. Ist der Blutdruck bis zu einem niedrigeren Stande wieder abgefallen, so werden die angeführten Organe wieder roth. Die Beobachtung des Ausflusses aus den Venen dieser Körperstellen lebrt des Weiteren, dass in dem Momente, in welchem der Blutdruck steigt, der Ausfluss oft verstärkt wird. Hat aher der Druck sein Maximum erreicht, dann nimint der Ausfluss bedeutend $a b$ und bleibt so lange gering, als der Druck hoch steht. Vorausgesetzt nun, dass alle diese Venen in das Gebiet der von Gärtner und Wagner benutzten Vene einmünden würden, so würde der Ausfluss bald nach der Extractinjection durch den verstärkten Zufluss aus dem äusseren Gebiete zwar eine Vermehrung erfahren; auf der Höhe des Blutdrucksmaximums aber, wenn sich der Zufluss verringert, müsste, wenn sich die Gehirngefässe nicht ändern würden, der Ausfluss unbedingt eine Abnahme erfahren. Das geschieht aber nicht; der Abfluss ist beim Maximum des Blutdruckes am mächtigsten und bleibt es auch, solange der Druck ein hoher ist, also zu einer Zeit, wo im äusseren Schädelgebiete die Gefässe contrahirt sind. Es muss somit der Blutstrom durch das Gehirn ein stärkerer geworden sein, und zwar ist derselbe darum verstärkt, weil, wie die directe Besichtigung des Gehirns lehrt, die Blutgefässe dilatirt worden sind. Sollte Fuchs etwa glauben, dass der venöse Strom durch das Retinalblut mächtiger geworden ist, so braucht er nur die Bulbi zu exstirpiren, und er wird trotzdem eine Vermehrung des Ausflusses constatiren können.

Ich binde in jüngster Zeit, wenn es nicht darauf ankommt, das Schädeldach intact zu erhalten, die Ausflusscanüle in den Sinus falciformis major ein. Die Methode ist weniger zeitraubend, und wenn die Canüle von der Dicke ist, dass sie den Sinus nicht ganz verlegt, so kann sie nach Belieben geöffnet und geschlosseu werden, ohne dass dadurch der Kreislauf des Gehirns wesentlich geändert wird.

1) Ueber die Einwirkung des Nebennierensaftes auf den Blutkreisianf. Wiener medic. Blätter $1896 \mathrm{Nr}$. 15-21. 
In dem Sinne der angefübrten Versuche muss aber die Forderung gestellt werden, dass man sich eines genügend wirksamen Nebennierenextractes bedient, besonders aber dann, wenn man die Ausflussgeschwindigkeit an einer Vene (Iugularis externa) prüft, die Blut aus dem Gehirne und anderen Körpergebieten enthält, wie das in den Versuchen von Fr. $\mathrm{Pick}^{1}$ ) geschehen ist. Ist die Steigerung des Blutdruckes in Folge der Extractinjection eine geringe, so ist es klar, dass dann eine mässige Verstärkung des Ausflusses aus dem Gehirne und eine Schwächung jenes aus den Nebengebieten interferiren können. Von diesem Standpunkte aus ist die Angabe von Pick, dass die Ausflussmenge aus der Iugularis nach der Extractinjection zur Zeit des Blutdruckmaximums dieselbe ist, wie zur Zeit des niederen Druckes zu beurtheilen, denn der mittlere Blutdruck nach der Injection betrug nach dem von Pick beigelegten Protokolle das eine Mal nur $143 \mathrm{~mm}$, ein anderes Mal $129 \mathrm{~mm}$.

Fuchs bringt auch an meiner historischen Darstellung der hier discutirten Fragen Correcturen an. Der Autor behauptet, dass Knoll bereits im Jahre $1888^{2}$ ) angegeben hätte, „dass Blutgerinnsel allein einen Gehirnprolaps hervorzurufen im Stande seien". Demzufolge hätte Knoll jenen Gehirnvorfall, den ich als Druckprolaps bezeichnet habe, vor mir gesehen. Jene Stelle in der Abhandlung Knoll's lautet: „In acht Fällen ${ }^{3}$ ) ging das Versuchsthier unter rascher, bis zum Athmungsstillstande zunehmender Verlangsamung und Abflachung der Athmung, bei deutlicher Hervordrängung der Oblongata aus dem Foramen obturatum zu Grunde. Die Section erwies, dass es in diesen Fällen zu einer starken Blutung und zur Bildung eines massigen Blutgerinnsels an der ventralen Seite der Oblongata gekommen war, wodurch die letztere in das Foramen obturatum hineingedrängt wurde." Wo ist denn hier von einem Gehirnprolaps die Rede? Oder ist es etwa gestattet, das in dem eröffneten Wirbelcanale freiliegende Rückenmark mit einer entblössten Area des von einer starren Wand umgebenen Gehirns gleichzusetzen? In dem Versuche Knoll's wird die Oblongata, somit das verletzte Organ, herausgedrängt, in meinem Versuche ist aber das herausgedrückte Organ unverletzt. Der citirte Befund weist eigentlich nur auf die

1) Archiv für experimentelle Pathologie und Pharmakologie Bd. 42. 1899.

2) Sitzungsberichte der k. Akad. d. Wissensch. Bd. 97. 1888.

3) Es handelt sich um mediane Spaltung der Oblongata bei Kaninchen. 
von alters her gemachte Beobachtung hin, dass ein Organ, wenn es angeschnitten wird, bluten und durch das Extravasat aus seiner Lage gebracht werden kann. Es könnte demnach Jedermann, der ein Hämatom gesehen, mit demselben Rechte wie Knoll und Fuchs behaupten, er habe den Druckprolaps des Gehirns vor mir beobachtet.

Die oben erwähnte Beobachtung, dass aus einer entblössten Hirnpartie der Liquor hervorsickert, wenn durch die Injection des Nebennierenextractes der Blutdruck erhöht und das Gehirn hyperämisch wird, rückte die Frage nach der Bildung desselben bei intactem Cranium in die Nähe.

Mit der Frage nach der Secretion des Liquors beschäftigten sich schon die Untersuchungen von $H$. Falkenheim und

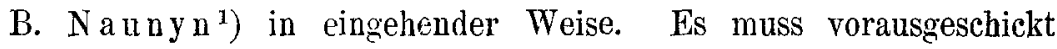
werden, dass die genannten Forscher die Cerebrospinalflüssigkeit als ein "specifisches Secret" ansehen.

Falkenbeim und Nauny haben in der Gegend der unteren Lendenwirbel subarachnoidal einen Gummischlauch eingeführt und diesen mit einer Messpipette in Verbindung gebracht. Die Ergebnisse dieser Versuche sind in Bezug auf die Einwirkung des arteriellen Blutdruckes auf die Secretion die folgenden: „Es führt jede Steigerung des arteriellen Blutdruckes (Unterbindung der Aorta thoracica und Strychninvergiftung) zu einer vorübergehenden Beschleunigung des Ausflusses der Cerebrospinalflüssigkeit, da der Druck in dieser mit dem Blutdrucke steigt. Doch gleicht sich diese vorübergehende Beschleunigung des Ausflusses durch eine schnell folgende Verlangsamung desselben aus; wenn man irgend grössere Zeiträume - über 15 Minuten - mit einander vergleicht, so findet man bei selbst sehr bedeutenden und anhaltenden Steigerungen des arteriellen Druckes keine Steigerung der Secretion." "Hingegen wurde die Secretion durch (intravenöse) Injection grösserer Mengen von physiologischer Kochsalzlösung erheblich (um 50\% und mehr) gesteigert."

Es kann nicht bezweifelt werlen, dass die eben beschriebene Methode den an sie gestellten Forderungen bessere Dienste geleistet hat, als die Einführung von Canülen durch das Schädeldach, welche

1) Archiv fur experimentelle Pathologie und Pharmakologie Bd. 22. 1887. 
auch meinen Erfahrungen zufolge sehr oft durch das sich eindrängende Gehirn verstopft werden. Zur Lösung der Frage nach der Bildung des Liquors langt aber diese Methode nicht hin. Denn die Aenderungen der Ausflussmenge sind unter Anwendung dieser Arbeitsweise einer dreifachen Deutung fähig. Eine Steigerung des Ausflusses könnte durch Volumsvermehrung des Gehirnes und dadurch bedingte Verdrängung des Liquors oder durch vermehrte Neubildung oder durch verhinderte Resorption desselben, das Sinken der Ausflussmenge ;durch Volumsabnahme des Gehirns oder durch verminderte Bildung des Liquors oder durch seine Resorption verursacht sein. Ich musste mir, da ich die Einwirkung der Blutüberfüllung des Gehirnes, also des erhöhten Blutdruckes kennen lernen wollte, andere Untersuchungsmittel zu Nutze machen.

Gelegentlich von Versuchen, welche ich über die Ausscheidung des Speichels aus der Glandula submaxillaris an curaresirten Hunden ausgefuhrt habe, erfuhr ich, dass man bei Anwendung von längeren, graduirten Röhren Beobachtungen machen kann, die beim Gebrauche von kurzen Ausflusseanülen dem Beobachter leicht, entgehen können. Ich habe nämlich die Walınehmung gemacht - auf Details gehe ich hier nicht ein -, dass der Speichel bei stark curaresirten Hunden nach wiederholten Reizungen der Chorda von der Drüse nur scheinbar secernirt wird, denn die an die Röhre abgegebene Secretmenge geht nach Sistirung der Reizung theilweise in die Drüse zurück. Es handelt sich somit hier nur um eine Verdrängung des Secretes. Diese Erfahrung bewog mich, auch bei den zu besprechenden Versuchen - bei denen ebenfalls eine Verdrängung des Liquors in Anschlag kommt - lange Röhren an Stelle der Ausflusscanülen zu verwenden. $\mathrm{Da}$ aber einerseits der Liquordruck ein geringer ist und andererseits die in die Röhre eintretende Menge desselben sich als recht beträchtlich erwies, musste die Röhre in schneckenförmige, in einer horizontalen Ebene gelegene Windungen auslaufen, welche die Versuche bedeutend erschwerten. Dies bewog mich, ohne mich des Vorzugs, der den graduirten Röhren zukommt, zu begeben, eine genügend lange, aber gerade Messröhre durch die Membrana obturatoria posterior in den Liquorraum einzuführen und den Liquor in derselben ansteigen zu lassen. Die mitzutheilenden Versuche werden ergeben, dass der durch diese Methode eingeführte Fehler für die Beurtheilung der Versuchsergebnisse ohne grossen Belang ist.

Ferner war es unausweichlich nothwendig, gleichzeitig den Blut- 
druck in der Aorta zu messen. Denn die Verdrängung des Liquors erfolgt ja durch eine Volumszunahme des Gehirns und diese wieder durch den Anstieg des Blutdruckes. Nur die Controle des jeweilig herrschenden Blutdruckes ermöglicht es, die Verdrängung von einer eventuellen Neubildung des Liquors zu unterscheiden. Wird, um nur ein concretes Beispiel anzuführen, der N. ischiadicus central gereizt, so nimmt das Gehirn an Volumen zu und der Liquor schreitet in der Röhre vor. Nach Unterbrechung der Reizung wird die Liquorsäule zwar kleiner, aber oft kann man die Wahrnehmung machen, dass dieselbe trotzdem um etwas länger als vor der Reizung ist. Ohne gleichzeitige Blutdruckverzeichnung ist eine auch nur annähernd richtige Beurtheilung dieser Erscheinung nicht möglich, denn der Zuwachs an Liquor könnte dadurch bedingt sein, dass der Blutdruck trotz der Sistirung der Reizung dennoch zu seinem früheren Stande noch nicht vollständig zurückgekehrt ist, oder dass dies zwar geschehen, aber dass die Hirngefässe sich noch nicht genügend entleert haben. Der Zuwachs könnte aber auch durch Neubildung von Liquor oder durch verhinderten Abfluss desselben hervorgerufen sein.

Die erste Reihe von Versuchen wurde an curaresirten Hunden mit beiderseits durchschnittenen Vagosympathici nach Entleerung der Cerehrospinalflüssigkeit ausgeführt. Der letztere Eingriff geschah in der Weise, dass das Thier nach Freilegung der Membrana obturatoria - die Blutgefässe müssen auf das Sorgfältigste unterbunden werden - mit dem Kopfe nach unten aufgehängt und durch die geschlitzte Membran eine feine Canüle eingeführt worden ist. Wenn der Liquor zi tropfen aufgehört hatte, wurde Bauch und Brust rhythmisch gedrückt und dadurch noch einige Tropfen herausgedrängt. Es gelingt auf diese Weise, den grössten Theil, aber keineswegs die ganze Cerebrospinalflüssigkeit zur Entleerung zu bringen. Hierauf wird das Thier in die Bauchlage gebracht, der feine Einschnitt in der Membrana obturatoria entsprechend der Dicke der zu beschreibenden Glasröhre erweitert und diese eingeführt. Dieselbe ist 40 Centimeter lang, an beiden Enden offen und knapp vor dem unteren, in den Wirbelcanal einzuführenden Ende derart konisch zugespitzt, dass das 4,5 Millimeter im Durchmesser messende Lumen an dieser Stelle um einen Millimeter kleiner ist. In einer Entfernung von drei Millimetern vom unteren Röhrenende sind zwei Seitenfenster - fünf Millimeter hoch und drei Millimeter breit einander gegenüber angebracht. Dem Liquor wird somit die Möglich- 
keit geboten, durch drei Oeffnungen in die Glasröhre einzudringen. Die Glasröhre ist graduirt, der Zwischenraum zwischen je zwei Theilstrichen beträgt drei bis vier Millimeter.

Die beschriebene Canüle ist für Hunde mittlerer Grösse (sechs bis sieben Kilo) bestimmt, bei grösseren Thieren erscheint es zweckentsprechender, eine Canüle von grösserer Länge und grösserem Kaliber zu verwenden. Dass die angegebenen Dimensionsverhältnisse nicht streng eingehalten werden müssen, brauche ich wohl nicht des Näheren auseinanderzusetzen; nothwendig ist nur, dass die Entfernung der fensterartigen Oeffnungen vom unteren Ende nicht weit von drei Millimetern differirt und das untere offene Ende konisch und stumpf auslauft. Derlei Röhren können leicht improvisirt werden.

Mit ihrem konischen Ende wird die Glascanüle durch die Oeffnung der Membrana obturatoria so tief eingefüht, bis die beiden Seitenfenster unterhalb derselben zu liegen kommen. Dabei ist darauf zu achten, dass der Schlitz in der Membran kleiner gemacht wird, als die Röhre unten dick ist, damit die letztere in der Oeffnung wasserdicht sitzt und genügend fixirt wird. Sodann wird die Canüle in einer dorsalwärts etwas geneigten Lage befestigt. Ist nun im Verlaufe des Versuches der Liquor in ihr aufoestiegen, so wird man gewahr werden, dass sich derselbe in grossen Excursionen bewegt, von denen - wie allgemein bekannt ist - die aufsteigende mit dem künstlichen In-, die absteigende mit dem künstlichen Exspirium zusammenfällt; die Pulsationen treten am deutlichsten in Erscheinung, wenn man die künstliche Respiration für einen Augenblick unterbricht. Dem Herzschlage gegenüber erscheint die Liquorsäule ungemein empfindlich. Zu einer Zeit, in welcher weder das Quecksilbermanometer, noch die äussere Palpation, noch die Auscultation einen Herzschlag erkenner lässt, kann man am Liquor noch systolische, allerdings sehr geringe Schwankungen wahrnehmen ${ }^{1}$ ). Manchmal kann es sich ereignen, dass der sonst bei gelungener Einführung wasserklare Liquor vom Blute etwas gefärbt erscheint, ein Umstand, der von keiner grossen Bedeutung ist, wenn die rothe Färbung im Laufe des Versuches nicht zu-, sondern abnimmt. Im entgegengesetzten Falle ist der Versuch für verfehlt anzusehen. Die vor-

1) Möglicher Weise handelt es sich hier, wie bei den Untersuchungen P. Ziegler's (Archiv für klinische Chirurgie Bd. 53, 1896) um Contractionen des rechten Vorhofes. 
Ueber den Einfluss des hohen Blutdruckes auf die Neubildung ete. 389

liegende Abhandlung bezieht sich nur auf jene Fälle, in welchen der Liquor vollständig wasserklar war.

$\mathrm{Zu}$ bemerken ist noch, dass die Thiere gut curaresirt werden müssen.

Da in den folgenden Versuchen der Blutdruck durch Injection von Nebennierenextract erhöht wurde, sind, wie schon mitgetheilt worden ist, zuvor die Vagi durchtrennt worden, da durch die im Gefolge der Injection auftretende Vagusreizung der Anstieg des Blutdruckes gemindert werden könnte.

Der Extract wird kurz vor dem Versuche mittelst physiologischer Kochsalzlösung bereitet ${ }^{1}$ ) und in die V. femoralis injicirt, wobei jeder Zug oder Druck an der Extremität und dem Bauche zu vermeiden ist.

Behufs Fixirung der zu beobachtenden Erscheinungen wurde in der Weise vorgegangen, dass der Liquorstand laut zu Protokoll dictirt und gleichzeitig der Moment der Ablesung auf der Blutdruckscurve markirt wurde. Die Marken am Kymogramm wurden nach dem Versuche mit fortlaufenden Zahlen bezeichnet und der betreffende Liquorstand aus dem Protokolle über den Marken eingetragen. Die Ablesung des Liquorstandes geschah immer am Ende der künstlichen Inspiration, wenn der Liquor in seiner inspiratorischen Excursion am höchsten stand.

\section{Versuch $\mathbf{I}$.}

Hund, curaresirt bis zur vollständigen Unbeweglichkeit, künstliche Ventilation, beide Vagi durchschnitten. Entfernung des Liquors, Einführung einer Injectionscanüle in die Vena femoralis, Verbindung der contra-lateralen Arteria cruralis mit dem Kymograph und Einführung der Messröhre durch die Membr. obturatoria posterior. Der Blutdruck beträgt $100 \mathrm{~mm} \mathrm{Hg}$, die Röhre ist leer, auch an ihrem Ende ist keine Flüssigkeit wahrzunehmen.

Der weitere Ablauf des Versuches ist der folgende ${ }^{2}$ ):

\begin{tabular}{r|c|c|c}
\hline \hline $\begin{array}{c}\text { Blut- } \\
\text { druck }\end{array}$ & Höhe der Liquorsäule & $\begin{array}{c}\text { Blut- } \\
\text { druck }\end{array}$ & Höhe der Liquorsäule \\
\hline 100 & 0 & 90 & 0 \\
96 & 0 & 88 & 0 \\
98 & 0 & 99 & 0
\end{tabular}

1) Experimentelle Untersuchungen über die Bildung des Liquor cerebrospinalis. Arch. f. d. ges. Physiologie Bd. 76 S. 208. 1899.

2) Der Raumersparniss wegen können leider die erzielten Resultate nicht an der Hand von Curven erläutert werden. 


\begin{tabular}{|c|c|c|c|}
\hline $\begin{array}{l}\text { Blut- } \\
\text { druck }\end{array}$ & Höhe der Liquorsäule & $\begin{array}{l}\text { Blut- } \\
\text { druck }\end{array}$ & Höhe der Liquorsäule \\
\hline \multirow{2}{*}{\multicolumn{2}{|c|}{$\begin{array}{l}\text { Injection von } 0,5 \mathrm{ccm} \text { des frisch be- } \\
\text { reiteten Extractes in die Vena femoral. }\end{array}$}} & 226 & 36 \\
\hline & & 220 & 34 \\
\hline 230 & 0 & $\begin{array}{l}204 \\
196\end{array}$ & $\begin{array}{l}32 \\
30\end{array}$ \\
\hline 240 & $\begin{array}{c}0 \\
0\end{array}$ & 174 & 28 \\
\hline 260 & Am Ende der Röhre er- & 150 & 22 \\
\hline & mit Luftblasen & 130 & 16 \\
\hline 270 & 10 & $\begin{array}{l}110 \\
100\end{array}$ & $\begin{array}{l}12 \\
10\end{array}$ \\
\hline & Die Luftblasen steigen empor & $\begin{array}{r}100 \\
94\end{array}$ & $\begin{array}{r}10 \\
5\end{array}$ \\
\hline 270 & $\begin{array}{l}\text { und verschwinden } \\
11\end{array}$ & 88 & 0 \\
\hline 268 & 12 & 80 & 0 \\
\hline 260 & 13 & \multicolumn{2}{|c|}{ Injection von $2,0 \mathrm{ccm}$} \\
\hline 256 & 14 & 130 & 10 \\
\hline 244 & 14 & & Keine Luftblasen \\
\hline 230 & 13 & 170 & 20 \\
\hline 224 & $\begin{array}{r}10 \\
5\end{array}$ & 210 & 31 \\
\hline $\begin{array}{l}216 \\
210\end{array}$ & & 230 & 33 \\
\hline 80 & 0 & $\begin{array}{l}254 \\
268\end{array}$ & $\begin{array}{l}40 \\
46\end{array}$ \\
\hline \multicolumn{2}{|c|}{ Injection von $1,0 \mathrm{ccm}$ des Extractes } & 272 & 50 \\
\hline 150 & 0 & 280 & 52 \\
\hline 230 & 2 & $\begin{array}{l}276 \\
274\end{array}$ & 54 \\
\hline 250 & Wenice Lufthläschen & $\begin{array}{l}274 \\
270\end{array}$ & $\begin{array}{l}56 \\
58\end{array}$ \\
\hline 260 & $\begin{array}{l}\text { Wenige Luttbläschen } \\
10\end{array}$ & 254 & 57 \\
\hline & Die Mehrzahl der Luftblasen & 230 & 56 \\
\hline & ist verschwunden & 200 & $\begin{array}{l}56 \\
52\end{array}$ \\
\hline $\begin{array}{l}270 \\
270\end{array}$ & $\begin{array}{r}18 \\
20\end{array}$ & 170 & 50 \\
\hline 266 & $\begin{array}{l}20 \\
22\end{array}$ & 140 & 40 \\
\hline 260 & 24 & 110 & 30 \\
\hline 930 & $\begin{array}{l}24 \\
22\end{array}$ & 94 & 20 \\
\hline 224 & 20 & 86 & 19 \\
\hline 210 & 19 & 80 & 17 \\
\hline 196 & 16 & \multicolumn{2}{|c|}{ Injection von $2,5 \mathrm{ccm}$} \\
\hline 178 & 14 & & 20 \\
\hline 156 & 10 & 136 & 30 \\
\hline 144 & 8 & 180 & 40 \\
\hline 134 & 0 & 216 & 50 \\
\hline $\begin{array}{r}104 \\
80\end{array}$ & 0 & 225 & 53 \\
\hline 76 & 0 & 240 & 60 \\
\hline \multicolumn{2}{|c|}{ Injection von $1,5 \mathrm{ccm}$ des Extractes } & 250 & 68 \\
\hline 130 & 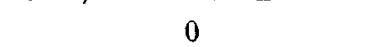 & 246 & 70 \\
\hline 190 & $\begin{array}{l}0 \\
5\end{array}$ & 240 & 71 \\
\hline & Ohne Iuftblasen & 220 & 71 \\
\hline 230 & 17 . & 200 & 71 \\
\hline & $\begin{array}{l}\text { Eine Luftblase steigt empor } \\
\text { und verschwindet }\end{array}$ & 190 & 71 \\
\hline 240 & $\begin{array}{c}20 \\
20\end{array}$ & 144 & 60 \\
\hline 260 & 30 & 120 & 50 \\
\hline 260 & 34 & $\begin{array}{r}100 \\
80\end{array}$ & 40 \\
\hline 250 & 38 & 76 & $\begin{array}{l}35 \\
32\end{array}$ \\
\hline $\begin{array}{l}246 \\
230\end{array}$ & $\begin{array}{l}38 \\
37\end{array}$ & & jection von $3,0 \mathrm{ccm}$ \\
\hline
\end{tabular}


Ueber den Einfluss des hohen Blutdruckes auf die Neubildung etc.

\begin{tabular}{c|c|c|c}
\hline $\begin{array}{c}\text { Blut- } \\
\text { druck }\end{array}$ & Höhe der Liquorsàule & $\begin{array}{c}\text { Blut- } \\
\text { druck }\end{array}$ & Höhe der Liquorsänle \\
\hline 106 & 40 & 208 & 87 \\
140 & 50 & 176 & 80 \\
190 & 60 & 150 & 70 \\
230 & 70 & 130 & 60 \\
270 & 80 & 104 & 50 \\
260 & 86 & 94 & 47 \\
250 & 90 & 82 & 40 \\
240 & 91 & 80 & 39 \\
230 & 91 & 70 & 30
\end{tabular}

Ein vergleichender Ueberblick der hier mitgetheilten Zahlenreihen lehrt, dass mit dem Ansteigen des Blutdruckes auch der Liquor in der Canüle ansteigt und mit dem Sinken des ersteren auch der letztere fällt, dass aber dieses Verhältniss unter gewissen Bedingungen nicht vorhanden ist und, wenn es vorhanden ist, keinen genauen Parallelismus aufweist.

Es ergibt sich dies aus dem Folgenden:

1. Vor der ersten Injection blieb die Canüle bei Schwankungen des Blutdruckes zwischen 88-100 leer. Es ist somit nicht zu bezweifeln, dass bei einem noch niedrigeren Drucke etwa von 80 die Röhre um so weniger Liquor enthalten würde. Untersucht man nun, welche Liquormengen dem angeführten Blutdrucke nach den einzelnen Injectionen entsprechen, so erhalten wir die nachstehenden Zahlen:

$\begin{array}{cccc} & & \text { Blutdruck } & \begin{array}{c}\text { Liquormenge } \\ \text { in Theilstrichen }\end{array} \\ \text { Vor der ersten Injection } & 80 & 0 \\ " \text { " zweiten " } & 80 & 0 \\ " \text { "dritten " } & 80 & 0 \\ " \text { "vierten " } & 80 & 0 \\ \text { " fünften " } & 80 & 17 \\ \text { Nach " lechsten " letzten " } & 80 & 35 \\ \end{array}$

Nimmt man somit $80 \mathrm{~mm}$ als den Ausgangsdruck an, so folgt aus dem Gesagten, dass mit den Injectionen bei demselben Ausgangsdrucke die Liquormengen in der Röhre wachsen.

2. Zur Zeit, in welcher der Blutdruck sein Maximum eben erreicht hat, verhält sich die Höhe der Liquorsảule wie folgt:

$270 \mathrm{~mm}=10$ Theilstriche nach der ersten Injection

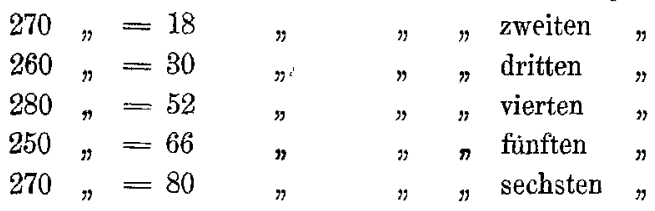

Die erreichten Blutdruckmaxima schwanken somit auf und ab, die Hohe der Liquorsäule steigt aber ohne Rücksicht auf diese Schwankungen mit jeder folgenden Injection an und zwar von 10 auf 80 Theilstriche. 
3. Ein weiterer Vergleich ergibt, dass das Liquormaximum nicht mit dem Blutdruckmaximum zeitlich zusammenfallt. Das erstere stellt sich erst dann ein, wenn der Blutdruck schon im Absinken begriffen ist. Diese Schlussfolgerung gilt für alle sechs Injectionen, ich führe aber nur als Beispiel die entsprechenden Zahlenpaare nach der ersten Injection an. Der Blutdruck erreichte das Maximum von 270, die Liquorhöhe betrug 10 Theilstriche; während aber noch der Blutdruck dieses Maximum beibehält, erhebt sich der Liquor auf 11, und wenn dann später der Blutdruck auf 244 fällt, steigt der Liquor trotzdem auf 14 empor.

4. Die nach jeder Injection angegebenen Blutdruckshöhen lehren, dass der Blutdruck vorerst ansteigt und dann nach erreichtem Maximum wieder fällt. Man kann demnach eine auf- und eine absteigende Phase des Blutdruckes unterscheiden. Beachtet man nun die Liquormengen, welche anf einen bestimmten Blutdruckswerth - etwa $230 \mathrm{~mm}$-, aber nur in allen aufsteigenden Phasen entfallen, so erhält man die folgenden Zahlenpaare:

Nach der ersten Injection bei $230 \mathrm{~mm}=0$ Theilstriche

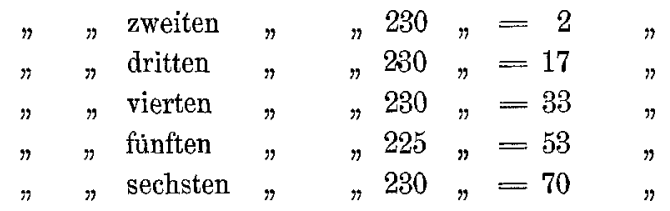

Die Höhe des Liquors nimmt demgemäss bei einem und demselben Blutdrucke in der Phase seines Aufstieges von Injection zu Injection zu.

5. Die Höhe des Liquors nimmt aber auch - wie die nachstehenden Zahlen lehren - bei 'einem und demselben Blutdrucke in den Phasen des sinkenden Blutdruckes mit jeder Injection zu.

Nach der ersten Injection bei einem Blutdrucke von $230 \mathrm{~mm}=13$ Theilstriche

\begin{tabular}{|c|c|c|c|c|c|c|c|c|c|}
\hline & $"$ zweiten & $"$ & $"$ & $"$ & $"$ & $"$ & 230 & $n$ & $=22$ \\
\hline & $n$ dritten & $n$ & $"$ & $"$ & $\eta$ & $"$ & 230 & $"$ & $=37$ \\
\hline & $"$ vierten & $"$ & $"$ & $"$ & $"$ & $"$ & 230 & $"$ & $=56$ \\
\hline & $"$ fünften & $"$ & $"$ & $"$ & $"$ & $"$ & 220 & $"$ & $\begin{array}{l}=71 \\
-91\end{array}$ \\
\hline & $"$ sechsten & $"$ & $"$ & $"$ & $n$ & $"$ & & $n$ & $=91$ \\
\hline
\end{tabular}

6. Vergleicht man die unter 4 und 5 angegebenen und zu einer und derselben Injection gehörigen Liquormengen, so wird man finden, dass die während der Phase des abfallenden Blutdruckes notirten Liquorzahlen grösser sind als jene während des ansteigenden Blutdruckes. So beträgt bei dem Blutdrucke von 230 nach der ersten Injection in der Phase des Blutdruckanstieges die Liquormenge 0 Theilstriche, während sie bei demselben Drucke in der Phase des fallenden Blutdruckes mit 13 bemessen erscheint.

Der eben beschriebene Versuch wird nur als Repräsentant mehrerer unter denselben Modalitäten und mit demselben Erfolge ausgeführter Experimente hier angeführt. Bemerkenswerthe Abweichungen ergeben sich nur nach der Richtung hin, dass in einigen Versuchen die der dritten Injection nachfolgenden weiteren Einspritzungen die 
Ueber den Einfluss des hohen Blutdruckes auf die Neubildung ete. 393

sub 1-6 angeführten Zahlenverhältnisse nicht klar zu erkennen gaben. Ich werde auf diesen Umstand noch zurückkommen.

Dass auch unwesentliche Modificationen in der Höhe des Blutdruckes und der Liquormenge eintreten können, ist schon durch das individuelle Verhalten der Thiere dem Extracte gegenüber begründet. So können mit gleichen Extractdosen Blutdruckswerthe von 250-330 und mehr erzielt werden und dementsprechend kann auch der Liquor verschieden hoch ansteigen.

Auch die Art, bis zu welchem Grade der Liquor vor dem Versuche entleert worden ist, scheint das Ergebniss, allerdings nur in unwichtigen Punkten, zu beeinflussen. So kann es sich ereignen, dass nach der ersten Injection noch kein Liquor in die Röhre eintritt, sondern erst nach der zweiten in derselben emporsteigt. Ferner sei noch erwähnt, dass, wenn statt des Extractes dieselben Mengen von physiologischer Kochsalzlösung injicirt werden, die Messröhre am Schlusse des Versuches leer bleibt oder nur eine geringe Menge von Liquor - etwa fünf Theilstriche - enthält.

\section{Versuch.}

Hund, curaresirt bis zur vollständigen Immobilität, künstliche Ventilation, beide Vagosympathici durchtrennt. Der Liquor wird nicht entleert. Einführung einer Injectionscanüle in die rechte Vena femoralis und der Messröhre durch die Membrana obturatoria unter die Dura. Verbindung der linken Art. cruralis mit dem Kymograph. Es sollen hier nur die wichtigeren Zahlenpaare angeführt werden:

\begin{tabular}{|c|c|c|c|}
\hline $\begin{array}{l}\text { Blut- } \\
\text { druck }\end{array}$ & Höhe der Liquorsäule & $\begin{array}{l}\text { Blut- } \\
\text { druck }\end{array}$ & Höhe der Liquorsäule \\
\hline 100 & 15 & 380 & 97 \\
\hline $\begin{array}{l}\text { Injection } \\
\text { Nebennie }\end{array}$ & $\begin{array}{l}1 \mathrm{ccm} \text { des frischbereiteten } \\
\text { tractes in die Vena femor. }\end{array}$ & : & : \\
\hline $\begin{array}{l}300 \\
310 \\
320 \\
330 \\
332 \\
333 \\
334 \\
335 \\
335 \\
335 \\
334 \\
333 \\
332 \\
332 \\
331\end{array}$ & $\begin{array}{l}30 \\
40 \\
45 \\
48 \\
55 \\
60 \\
70 \\
80 \\
90 \\
91 \\
92 \\
93 \\
94 \\
95 \\
96\end{array}$ & $\begin{array}{l}260 \\
250 \\
240 \\
\cdot \\
\dot{1} \\
178 \\
120 \\
100 \\
\text { In } \\
160 \\
260 \\
288\end{array}$ & $\begin{array}{c}97 \\
96 \\
96 \\
\cdot \\
\dot{83} \\
42 \\
38 \\
\text { n von } 1 \text { ccm Extract } \\
50 \\
60 \\
70\end{array}$ \\
\hline
\end{tabular}




\begin{tabular}{|c|c|c|c|}
\hline $\begin{array}{l}\text { Blut- } \\
\text { druck }\end{array}$ & Höhe der Liquorsáule & $\begin{array}{l}\text { Blut- } \\
\text { druck }\end{array}$ & Höhe der Liquorsäule \\
\hline 300 & 90 & \multicolumn{2}{|c|}{ Injection von 2 ccm Extract } \\
\hline 310 & 120 & 160 & 50 \\
\hline 320 & 135 & 240 & 70 \\
\hline 325 & 136 & 260 & 90 \\
\hline 326 & 140 & 285 & 120 \\
\hline 325 & 140 & 300 & 130 \\
\hline . & . & 310 & 145 \\
\hline . & . & 320 & 150 \\
\hline . & 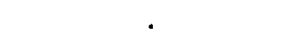 & 321 & 152 \\
\hline . & . & 323 & 156 \\
\hline & 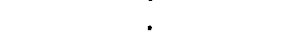 & 320 & 157 \\
\hline 280 & 140 & 319 & 160 \\
\hline 260 & 135 & . & - \\
\hline$\cdot$ & . & 260 & 160 \\
\hline $2 \dot{20}$ & $1 \dot{30} 0$ & 245 & 150 \\
\hline . & . & 203 & 95 \\
\hline & & 170 & 88 \\
\hline 120 & 95 & 140 & 87 \\
\hline 100 & 45 & 100 & 70 \\
\hline
\end{tabular}

Eine Analyse der vorliegenden Zahlen ergibt dasselbe Resultat wie im ersten Versuche, die Zahlen sind aber absolut höher, da die Cerebrospinalfüssigkeit nicht entleert worden ist. Bemerkenswerth ist das lange Beharren der Liquorsäule auf ihrem Maximum zu einer Zeit, als der Blutdruck schon bedeutend abgefallen war, und die bedeutenden Höhen des letzteren. Obwohl in diesem Versuche die Maxima des Blutdruckes mit jeder Injection geringer werden, wird trotzdem die Liquorsäule mit jeder Injection höher.

\section{Versuch.}

Es wird in derselben Weise wie im ersten Versuche experimentirt. Es sollen hier nur die wichtigsten Zahlen mitgetheilt werden.

\begin{tabular}{|c|c|c|c|}
\hline $\begin{array}{l}\text { Blut- } \\
\text { druck }\end{array}$ & Höhe der Liquorsäule & $\begin{array}{l}\text { Blut- } \\
\text { druck }\end{array}$ & Höhe der Liquorsäule \\
\hline 150 & 0 & 330 & 3 \\
\hline \multicolumn{2}{|c|}{$\begin{array}{l}\text { Während der nächsten zehn Minuten } \\
\text { schwankt der Blutdruck zwischen } 152 \\
\text { bis } 146 \text {, die Röhre bleibt leer. } \\
\text { Injection von } 1 \text { ccm des frisch bereiteten } \\
\text { Extractes }\end{array}$} & $\begin{array}{l}320 \\
318 \\
: \\
:\end{array}$ & $\begin{array}{l}4 \\
5 \\
. \\
\end{array}$ \\
\hline 190 & 0 & 156 & 2 \\
\hline 280 & $\stackrel{0}{0}$ & 140 & 0 fünf Minuton \\
\hline $\begin{array}{l}300 \\
320\end{array}$ & $\begin{array}{c}\text { Am Ende der Röhre Luft- } \\
\text { blasen mitetwasFlussigkeit } \\
2 \\
\text { Die Luftblasen steigen empor }\end{array}$ & $\begin{array}{l}\text { Wahreno } \\
\text { fallt der } \\
\text { Inje }\end{array}$ & $\begin{array}{l}\text { der folgenden fünf Minuten } \\
\text { Blutdruck auf } 126 \text {, die Röhre } \\
\text { bleibt leer. } \\
\text { ction von } 1 \mathrm{ccm} \text { Extract }\end{array}$ \\
\hline
\end{tabular}


Ueber den Einfluss des hohen Blutdruckes auf die Neubildung etc. 395

\begin{tabular}{|c|c|c|c|}
\hline $\begin{array}{l}\text { Blut- } \\
\text { druck }\end{array}$ & Hòhe der Liquorsäule & $\begin{array}{l}\text { Blut- } \\
\text { druck }\end{array}$ & Hòhe der Liquorsäule \\
\hline 280 & 2 & 268 & 38 \\
\hline 300 & 5 & 250 & 37 \\
\hline 320 & 10 & . & . \\
\hline 318 & 12 & . & . \\
\hline 315 & 14 & . & 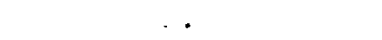 \\
\hline 300 & 16 & & \\
\hline . & . & 210 & Anf diecer Höhe bleiht der \\
\hline$\cdot$ & : & & Blutdruck und die Liquor- \\
\hline $2 \dot{20}$ & $\dot{12}$ & & $\begin{array}{l}\text { saule durch } 3 \text { Minuten un- } \\
\text { verändert stehen und fällt }\end{array}$ \\
\hline 130 & 8 & & dann in den nächsten $2 \mathrm{Mi}-$ \\
\hline Während & der folgenden 15 Minuten & 208 & $\begin{array}{l}\text { nuten auf } \\
10\end{array}$ \\
\hline & säule auf: & \multirow{2}{*}{\multicolumn{2}{|c|}{$\begin{array}{l}\text { Erötfnung des Thorax auf beiden Seiten } \\
\text { und Injection von } 2 \mathrm{ccm} \text { Extract }\end{array}$}} \\
\hline 119 & 6 & & \\
\hline \multicolumn{2}{|c|}{ Injection von $2 \mathrm{ccm}$ des Extractes } & 210 & 16 \\
\hline 220 & 14 & - & . \\
\hline - & . & & • \\
\hline - & & $2 \dot{7} 6$ & 36 \\
\hline 270 & 20 & 274 & 38 \\
\hline 275 & 30 & 264 & 40 \\
\hline 275 & 34 & 260 & 41 \\
\hline 272 & 36 & 268 & 40 \\
\hline
\end{tabular}

Abgesehen davon, dass auch dieser Versuch die oben unter 1-6 mitgetheilten Erfahrungen bestätigt, bleibt noch hervorzuheben, dass in den Pausen vor den Injectionen, welche absichtlich verlängert worden sind, die Liquormenge $=0$ war oder dass die Liquorsäule kleiner wurde. Erst unter der Einwirkung des Extractes fing dieselbe zu wachsen an. Endlich lehrt der Versuch, dass eine beiderseitige Eröffnung der Brusthöhle das Ansteigen der Liquorsäule nicht verhindert.

\section{Versuch.}

Präparation wie im Versuche I. Es werden fünf Injectionen des Extractes, bei welchen $12 \mathrm{~cm}$ zur Verwendung gekommen sind, mit dem schon bekannten Resultate ausgeführt. Der Stand des Blutdruckes und des Liquors vor der sechsten Injection ist der folgende:

\begin{tabular}{|c|c|c|c|}
\hline $\begin{array}{l}\text { Blut- } \\
\text { druck }\end{array}$ & Höhe der Liquorsäule & $\begin{array}{l}\text { Blut- } \\
\text { druck }\end{array}$ & Höhe der Liquorsäule \\
\hline 80 & 38 & 130 & 4.5 \\
\hline \multicolumn{2}{|c|}{ Sechste Injection: $3 \mathrm{ccm}$ des Extractes } & $\begin{array}{l}162 \\
190\end{array}$ & 50 \\
\hline 100 & 42 & 210 & 68 \\
\hline
\end{tabular}




\begin{tabular}{c|c|c|c}
\hline \hline Blutdruck & $\begin{array}{c}\text { Höhe } \\
\text { der Liquorsäule }\end{array}$ & Blutdruck & $\begin{array}{c}\text { Höhe } \\
\text { der Liquorsäule }\end{array}$ \\
\hline 230 & 70 & 210 & 81 \\
234 & 75 & 200 & 77 \\
240 & 80 & 172 & 70 \\
234 & 82 & 134 & 60 \\
232 & 81 & 80 & 55 \\
230 & 81 & Achte Injection: 4 ccm \\
225 & 80 & 90 & 57 \\
162 & 79 & 120 & 71 \\
130 & 67 & 195 & 72 \\
100 & 64 & 218 & 78 \\
80 & 55 & 218 & 78 \\
Siebente Injection: 3,5 ccm & 65 & 208 & 77 \\
98 & 70 & 170 & 69 \\
120 & 72 & 140 & 63 \\
196 & 80 & 135 & 60 \\
220 & 80 & 80 & 54 \\
220 & 81 & & \\
219 & & &
\end{tabular}

Ein Vergleich der Zahlenreihen lehrt, dass mit Ausnahme der sich zur Zeit der Blutdruckmaxima ergebenden Zahlenverhältnisse ein gewisser Parallelismus zwischen der Höhe des Blutdruckes und der Höhe der Liquorsäule unter den genannten Versuchsbedingungen zu Tage tritt. So entsprechen der Blutdruckshöhe von $80 \mathrm{~mm}$ in der Reihenfolge der Injectionen die nahezu gleichen Liquorhöhen von 55,55 und 54 . Es sinken ferner mit den Blutdruckmaximen auch die Liquormaxima. Ueberdies, und das ist das Auffallendere, differiren die letzteren nur in einem geringen Grade $(82,81,78)$.

\section{Versuch.}

Hund, curaresirt, künstliche Ventilation. Vagi durchschnitten. Rechte Cruralis verbunden mit dem Kymograph, durch die Membrana obturatoria die Messröhre eingeführt. Aussetzung der künstlichen Athmung.

\begin{tabular}{c|c|c|c}
\hline Blutdruck & $\begin{array}{c}\text { Hohe } \\
\text { der Liquorsaule }\end{array}$ & Blutdruck & $\begin{array}{c}\text { Höhe } \\
\text { der Liquorsäule }\end{array}$ \\
\hline & 17 & 182 & 29 \\
100 & 16 & 160 & 30 \\
90 & 15 & 140 & 31 \\
108 & 14 & 120 & 32 \\
110 & 15 & 108 & 32,5 \\
120 & 16 & 108 & 33 \\
140 & 20 & 90 & 32 \\
180 & 22 & 80 & 31 \\
182 & 24 & 48 & 28 \\
184 & 25 & 20 & 26 \\
186 & 26 & \multicolumn{2}{|c|}{ Tod des Thieres. } \\
184 & 27 & &
\end{tabular}


In Folge der Suspension der Athmung fällt die Liquorsäule vorerst $a b$, während der Blutdruck in derselben Zeit nach einem unbedeutenden Abfalle ansteigt. Der Blutdruck erhebt sich dann bis zu seinem Maximum von 186 an, auch die Liquorsäule wächst gleichzeitig heran, erreicht aber ihr Maximum von 33 Theilstrichen bedeutend später, indem der Blutdruck schon auf 108 gefallen ist.

Alle hier mitgetheilten Versuchsprotokolle beziehen sich auf Thiere, welche nach dem Versuche secirt worden und bei denen keine bemerkenswerthen Veränderungen am Hirn und Rückenmark, insbesondere aber keine Hämorrhagien oder Thrombosen gefunden worden sind. Bemerken möchte ich nur noch, dass das bald post mortem secirte Hundehirn auffallend stark glänzt, eine Erscheinung, die man auf einen stärkeren Feuchtigkeitsgehalt, vielleicht auf Oedem, desselben beziehen könnte. Ich habe mich aber überzeugt, dass der starke Glanz zu der gewöhnlichen Eigenthümlichkeit des Hundegehirns gehört.

\section{Discussion der Versuche.}

Die Literatur bezüglich des Kreislaufes im Gehirne enthält zahlreiche Angaben über das nähere Verhalten des cerebralen Gefässsystems, welche indicret aus Beobachtungen des Liquordruckes gewonnen worden sind. Ich verweise hier auf die Experimente von Moss $0^{1}$ ) an Schädeldefecten von Menschen, auf die Untersuchungen von $\mathrm{Knoll}{ }^{2}$ ), welcher an Thieren experimentirte, ferner an die Arbeiten von Falkenheim und $\mathrm{Nauny}^{8}$ ), P. Ziegler ${ }^{4}$ ) u. A. Es wurde hierbei an der Auffassung festgehalten, dass einerseits durch Dilatation der Gefässe von Hirn und Rückenmark der Liquor unter höheren Druck und andererseits durch Contraction der Gefässe unter geringeren Druck gesetzt wird. Die schärfste Fassung gewann dieses Princip in den Worten Mosso's (l. c. pag. 23): „Der Plethysmograph stellt eine Nachahmung des mit Flüssigkeit angefüllten Kastens, worin die Centralorgane des Nervensystems eingeschlossen sind, dar."

Es erscheint indessen dieses Princip durch ein Versuchsresultat Knoll's, ohne dass der Autor selbst dessen gewahr wurde, durch-

1) Ueber den Kreislauf des Blutes im menschlichen Gehirn. Leipzig 1881.

2) Sitzungsber. d. k. Akad. d. Wissenschaften in Wien Bd. 93. 1886.

3) l. c.

4) Archiv für klinische Chiurgie Bd. 53. 1896. 
brochen. Das Vertrauen in die Unwandelbarkeit der Beziehungen zwischen Gefässweite und Liquordruck hat aber so tiefe Wurzeln gefasst, dass man Erscheinungen, die jenem Principe widersprachen, trotzdem auf speculativem Wege unter dasselbe gebracht hat.

Knoll hat an curaresirten Hunden beobachtet, dass bei Dyspnoë der Liquordruck früher als der Blutdruck sich erhebt und länger auf seinem Maximum verharrt als der letztere und erklärt beide Erscheinungen getreu dem oben angeführten Standpunkte durch eine in Folge venöser Stauung eintretende Volumszunahme des Gehirns. Im ersteren Falle soll der Ausfluss des venösen Blutes durch die Athmungsaussetzung, im letzteren Falle durch die dyspnoëtische Reizung des Herzvagus bewirkt werden.

Falkenheim und Naunyn ${ }^{1}$ ) hingegen fanden, dass die Erhebung des Liquordruckes nach der Aussetzung der Athmung von einer Erniedrigung desselben eingeleitet wird. Ich habe diese initiale Senkung des Liquordruckes einige Male bei Anwendung meiner Methode gleichfalls beobachtet (Versuch IV). Ich will mich aber hier mit dieser Erscheinung nicht weiter beschäftigen und nur so viel erwähnen, dass es für das Verhalten des Liquors nicht gleichbedeutend ist, in welcher respiratorischen Phase die Athmung sistirt wird und $o b$ die an der Trachealeanüle angebrachte Seitencanüle offen gehalten oder geseblossen wird.

Die andere von Knoll gemachte Beobachtung, dass der maximale Liquordruck den maximalen Blutdruck überdauert, kann ich wohl als richtig bestätigen; aber der Deutung, dass dieselbe ein Effect der Reizung von hemmenden Herznerven ist, vermag ich nicht beizupflichten, denn, wie der Versuch $V$ lehrt, tritt jene Erscheinung auch nach bilateraler Vagotomie ein. Damit ist aber die Methode, aus dem Liquordrucke auf die Blutfülle des Gehirns und umgekehrt aus der letzteren auf den ersteren zu schliessen, als unverlässlich erkannt.

In Hinsicht auf diese Erfahrung sind wohl $\mathrm{Z}_{w}$ eifel gestattet über die Berechtigung, den Schädel und seinen Inhalt mit einem Plethysmographen $\mathrm{zu}$ analogisiren, und dieser Zweifel wird noch lebhafter, wenn man die Ergebnisse der oben mitgetheilten Versuche (I-V) mit dem Nebennierenextracte näher besieht.

Wäre das plethysmographische Princip in Bezug auf das Gehirn

1) l. c. 
vollgültig, dann müsste die Menge des in die graduirte Röhre gedrungenen Liquors nur als verdrängt angesehen werden. Diese Annahme würde aber mit den oben mitgetheilten Versuchsresultaten in Conflict gerathen.

Dass der Liquor thatsächlich von dem schwellenden Gehirne in die Röhre gedrängt wird, das unterliegt wohl keinem $Z$ weifel, denn schon die Excursionen der Liquorsäule in Folge der Schwankungen des intrathorakalen Druckes bei den respiratorischen Bewegungen und in Folge der Herzarbeit legen dies klar, und dass dies auch nach der Extractinjection der Fall ist, folgt aus dem Versuche IV, aus welchem ganz klar zu ersehen ist, dass, wenn die Blutdruckszahlen wachsen, auch die Liquorzahlen gleichsinnig grösser werden.

Dass aber der Liquor nu r verdrängt worden ist, das kann im Hinblicke der beim Versuche I unter 1-6 angeführten Deductionen nicht behauptet werden, denn das plethysmographische Princip wird durch dieselben direct widerlegt.

Gegen die unter 1 angefuhrte Folgerung, dass die Liquormengen bei demselben Ausgangsdrucke mit der Wiederholung der Extractinjection steigen, könnte möglicherweise eingewendet werden, dass dies durch in Gefolge der wiederholten Injectionen auftretende cerebrale Hämorrhagien oder Thrombosen oder vielleicht durch ein cerebrales Oedem bedingt ist, dass somit die Liquormengen darum grösser werden, weil mit jeder Injection die genannten pathologischen Veränderungen gesteigert werden und das Hirn auf sein früheres Volumen nicht mehr zurückkehrt. Demgegenüber ist zu bemerken, dass, wie schon angegeben worden ist, die Section keine pathologischen Veränderungen am Gehirne zu erkennen gab. Ausserdem müsste bei einem derart vergrösserten Gehirne das Liquormaximum vor dem Blutdruckmaximum eintreten, denn die Aufnahmefähigkeit des Gehirns für das Blut muss durch jene Processe verringert werden, und ad infinitum kann sich das Gehirn denn doch nicht vergrössern. Ich habe aber das Gegentheil von der vorausgesetzten Erscheinung beobachtet, das Liquormaximum folgte dem Blutdruckmaximum nach.

Es könnte aber noch ein anderer Einwand erhoben werden. Zugegeben, dass das Gehirn keine pathologischen Veränderungen erleidet, so könnte dem normalen Sectionsbefunde zum Trotze die Sachlage die folgende sein. Das Gehirn - könnte man sagen erreicht seine maximale Blutfulle nicht in dem Augenblicke, in welchem der Blutdruck seinen Gipfel erklommen hat, sondern später 
und befreit sich von dem überschüssigen Blute erst zu einer Zeit, in welcher der Blutdruck schon im Sinken begriffen ist, weil das Gehirn zur Anfüllung und Entleerung einer gewissen Zeit bedürfen könnte. Thatsächlich bieten diesem Einwande die sub 3-6 angeführten Folgerungen eine Stütze. Es sprechen aber gegen denselben die unter 1 und 2 mitgetheilten Zahlen. Es ist nicht einzusehen, warum die Liquormengen bei den in der Reihenfolge der Injectionen sich ergebenden Werthen des Ausgangsdruckes stejgen sollten, warum die Entleerung des Gehirns durch jede folgende Injection mehr verzögert werden sollte. Lehrt doch der Versuch IV, dass die Entleerung des Gehirns auch nach sechs bis acht Injectionen prompt erfolgt.

Aber ich verzichte auf dieses Argument, da es jenen Einwand nicht mit genügender Schärfe zu beseitigen vermag. Dagegen bieten die unter 2 angeführten Zahlen Verhältnisse, welche offenkundig gegen jenen Einwurf sprechen. Wenn die Blutdrucksmaxima nach den einzelnen Injectionen auf und ab schwanken und die Liquormengen trotzdem mit jeder folgenden Injection in die Höhe gehen, so ist jenem Einwurfe jede Berechtigung entzogen. Die verspätete Anfiullung und Entleerung des Gehirns kann nur bewirken, dass sich das Liquormaximum gegen den Blutdruck zeitlich verschiebt, dass das erstere dem letzteren nachhinkt, aber dieselbe vermag nicht das absolute Wachsen der Liquormaxima im Verlaufe der Injectionenserie zu erklären. Jenes Nachhinken ist nun thatsächlich zu erweisen, es kann demgemäss ein verspäteter Volumswechsel des Gehirns concedirt werden, aber es ist nicht gestattet, aus demselben abzuleiten, dass der ganze Ablauf der in den angeführten Versuchen beschriebenen Erscheinungen nur durch passive Verdrängung des Liquors hervorgerufen wird.

Wiewohl die verspätete Anfüllung und Entleerung des Gehirns dem Gesagten zufolge zur Erklärung der unter 1-6 angeführten Zahlenverhältnisse nicht hinreicht, hielt ich es doch für geboten, mich über diesen Punkt näher zu orientiren.

Die schon von Lorry gemachte Erfahrung, dass die in eine künstliche Schädelöffnung eingefügte und mit Flüssigkeit gefüllte Glasröhre die Athembewegungen synchronisch durch Hebung und Senkung des Flüssigkeitsniveaus anzeigt, und die von Mosso an Schädeldefekten von Menschen graphisch dargestellten Pulsationen, welche gleịchzeitig mit dem Herzschlage sich einstellen, lehren zur 
Genüge, dass das Volumen des Gehirns kleinere Schwankungen seines Blutgehaltes ohne merkliche Verspätung mitmacht. Trotzdem köunten die Verhältnisse bei grossen Variationen in der Blutfüllung, wie sie die Extractinjectionen hervorrufen, ganz anders liegen. Um hierüber Näheres zu erfahren, habe ich vorerst direct untersucht, wie rasch șich das entblösste Gehirn nach Extractinjectionen anfült und wieder entleert. Selbstverständlich musste der Liquor entfernt werden. $\mathrm{Zu}$ diesem Behufe wurde ein Hund curaresirt, die Vagi durchtrennt, der Liquor nach der früher angegebenen Weise entfernt, eine kreisförmige Fläche des Gehirns von etwa drei Centimer im Durchmesser durch Entfernung der Dura freigelegt und nun auf das Gehirn ein einarmiger Hebel gebracht.

Derselbe verzeichnete die respiratorischen und pulsatorischen Volumsschwankungen des Gehirns in einer prompten Weise. Unmittelbar hinter dem Hebelarme, aber doch so, dass derselbe sich ganz frei bewegen konnte, wurde eine Scala aufgestellt und die Excursionen des Hebels unter gleichzeitiger Controle des Blutdruckes beobachtet. Die Untersuchung ergab, dass, nachdem der Extract injicirt worden war, der Hebel das grösste Gehirnvolumen nach dem Maximum des Blutdruckes, sonit später, angezeigt und dass der Blutdruck früher als der Hebel zu sinken begonnen hat. Die zeitlichen Differenzen betrugen aber nur einige (vier bis acht) Secunden. Auch bei diesem Versuche wurden die Extractinjectionen wiederholt.

Wie nun unter 3 angegeben wurde, verspätet sich zwar das Liquormaximum gegen das Maximum des Blutdruckes. Das Stadium des hohen Blutdruckes nach Injection des Nebennierenextractes, von unwesentlichen Scbwankungen desselben abgesehen, hält aber 1-2 Minuten an und jener Theil der Curve, in welchem der Blutdruck schon im Sinken, der Liquor aber noch im Steigen begriffen ist, währt auch etwa 0,5 Minuten. Würde demgemäss das verzögerte Eintreffen des Liquormaximums von der Verspätung in der Anfüllung des Gehirns abhängen, müsste angenommen werden, dass die Zeit, welcher das Gehirn bis zur Erreichung seines maximalen Volumens bedarf, nach Minuten zu messen ist, und doch hat die Untersuchung des Gehirns mittelst des Hebels gelehrt, dass die Verspätung nur einige Secunden beträgt.

Injicirt man des Weiteren - unter Versuchsbedingungen, wie sie beim Experimente II angegeben sind - grössere Mengen des 
Extractes ${ }^{1}$ ), dann kann das Stadium des hohen Druckes oft bis auf 4 Minuten verlängert und der Abfall des Blutdruckes bedeutend verzögert werden. Die Zeit, welche von dem Eintritte des hohen Blutdruckes bis zum Eintritte des Liquormaximums verstreicht, beträgt dann oft $3-5$ Minuten.

Die sub 3 mitgetheiltè Beobachtung kann demnach durch eine verspätete Anfüllung allein nicht erklärt werden.

Aus den vorstehenden Erörterungen wird somit ersichtlich, dass das plethysmographische Princip auch unter der Annahme von pathologischen Veränderungen des Gehirns oder einer verspäteten Anfullung und Entleerung desselben zur Erklärung der mitgetheilten Beobachtungen nicht ausreicht. Es wäre demgemäss unberechtigt, die nach den Injectionen zu Tage tretenden Liquormengen als nur verdrängt anzusehen. Demzufolge erübrigt keine andere Erklärung: als die, dass die Liquormengen im Gefolge der Extractinjectionen verdrängt und neugebildet worden sind

Von diesem Gesichtspunkte aus lassen sich die oben geschilderten Erscheinungen (1-6) in der einfachsten und zwanglosesten Weise erklären. Wird der Liquor entleert (Versuch I und III), so bleibt die Messröhre leer; sowie aber der Extract injicirt wird, ziehen sich die kleineren und capillaren Gefässe in grossen Körpergebieten zusammen, der Blutdruck steigt und erweitert die Gefässe des Gehirns und wahrscheinlich auch die des Rückenmarkes. Durch die dilatirten Gefässe strömt nun eine grössere Menge von Blut, und gleichzeitig erscheint der Binnendruck derselben, wie die von mir ausgeführten Messungen des Blutdruckes im Circulus Willisii ergeben haben ${ }^{2}$ ), erhöht. Das Gehirn und vielleicht auch das Rückenmark werden voluminöser, drängen die Cerebrospinalflüssigkeit in die Röhre, und gleichzeitig wird neuer Liquor transsudirt. War die Entleerung des Liquors vor dem Versuche eine ausgiebige, so erscheint in der Mess. röhre keine Flussigkeit, im entgegengesetzten Falle können in die Canüle geringe Liquormengen mit Luft untermischt eindringen. Wenn jetzt der Extract zu wirken aufgehört hat, wird der allgemeine Blutdruck geringer, das Hirn und Ruckenmark kleiner, und die Liquorsäule zieht sich in die grösser gewordenen Liquorräume zurück. Dasselbe Spiel wiederholt sich bei der folgenden Injection. Da aber

1) Es wurden in der Reihenfolge der Injectionen 1, 2, 3,5, 4, 5, 6,5, $7 \mathrm{ccm}$ des Extractes verwendet.

2) P.flùger's Archiv Bd. 76. 
diese abermals eine Neubildung von Liquor im Gefolge hat, so wird dieser in grösserer Menge in die Röhre gedrängt werden. So kann es geschehen, dass bei den folgenden Injectionen durch die wiederholte Erzeugung von Liquor und seine Verdrängung die Röhre auch bei niederem Blutdrucke gefüllt bleibt, die Liquormaxima grösser werden und die zu Beginn des Versuches entleerten Liquorräume mit neuer Flüssigkeit gefüllt werden. Jetzt wird es begreiflich, warum die Liquorsäule, trotzdem das Gehirn sein maximales Volumen schon erreicht hat, weiter wächst. Durch die Gehirnschwellung werden die Liquorräume verengt, und der Liquor kann nicht in seinen natürlichen Recipienten dringen. Da aber die Neubildung der Flüssigkeit fortschreitet, steigt er in dem künstlichen Recipienten an und steigt so lange an, bis der natürliche Aufnahmeraum in Folge eines ausgiebigen Abfalles des Blutdruckes wieder geräumiger geworden ist. Des Weiteren wird nun die Erscheinung, dass gleichen Druckwerthen in der Phase des absteigenden Blutdruckes höhere Liquorzahlen entsprechen als in der des ansteigenden, einer Erkiärung zugänglieher. Die letztere Phase entwickelt sich ja vor dem Maximum, die erstere nach dem Maximum der Liquorbildung.

Endlich wird es auch klar, warum die Liquormaxima sich dem Blutdrucke gegenüber bis in das Stadium des sinkenden Blutdruckes verspäten. Durch die blosse Annahme einer verspäteten Anfüllung des Gehirns kann, wie sehon erörtert worden ist, diese Erfahrung nicht gedeutet werden, wohl aber, wenn wir neben der Verdrängung des Liquors noch eine Neubildung desselben in Anschlag bringen.

Man könnte sich vielleicht noch der Meinung hingeben, dass der Liquor zwar neugebildet werde, dass aber die Neubildung desselben nicht ein Effect der Extractinjectionen, das heisst der Gehirnhyperämie und des hohen Blutdruckes ist, sondern sich unabhängig von den Versuchseingriffen vollzieht. Zur Beseitigung dieses Einwandes genügt schon der Hinweis auf die im Versuche III gemachten Erfahrungen, dass, wenn vor einer jeden Injection eine längere Pause eingeführt wird, während derselben der Liquor mit dem Blutdrucke sinkt oder Blntiruck und Liquor auf ihrem Niveau verharren. Erst durch die Extractinjection wird ein neues Mehr von Liquor geschaffen. Es fällt mir aber trotzdem nicht bei, jede von den Injectionen unabhängige Liquorbildung zu negiren; dieselbe ist aber jedenfalls gegenüber dem durch den Extract bewirkten Plus eine geringe. 
Wenn wir uns das Vorstehende noch einmal vergegenwärtigen, so gelangen wir zu dem Schlusse, dass durch die Extractinjection Liquor neugebildet, transsudirt wird und dass der hohe Blutdruck und die daraus resultirende Hyperämie des Gehirns - warscheinlich auch die der Pia, bewiesen ist dies aber nicht - das veranlassende Moment der Neubildung des Liquors abgeben. Bei dem auffallenden Unterschiede in der chemischen Zusammensetzung der Cerebrospinalfüssigkeit und des Blutplasma ist die Annahme einer blossen Filtration ausgeschlossen. Möglicherweise wird das Filtrat von der Gefässwand auf eine bis jetzt unbekannte Weise beeinflusst. $\mathrm{Zu}$ Gunsten der Annahme, dass der Liquor ein Secret vorstellt, liegen, wie ich schon in einer früheren ${ }^{1}$ ) Publication erwähnt habe, keine Beobachtungen vor.

In Rücksicht auf die Injectionsversuche mit dem Nebennierenextracte ist, entsprechend den im Versuche IV gemachten Beobachtungen, noch zu erwähnen, dass die Neubildung des Liquors nach oft wiederholten Injectionen versiegt. Die im Versuche I noch nach der sechsten Injection beobachtete Liquorbildung gehört nicht zur Regel. Oft versiegt die Neubildung der Cerebrospinalflüssigkeit nach der vierten Injection. Es reichen aber für gewöhnlich schon drei Injectionen hin, um die hier unter $1-6$ mitgetheilten Facta klar zu sehen. Der Grund, warum oftmals wiederholte Injectionen zur Liquorbildung nicht mehr beitragen, war in einigen Fällen dadurch begründet, dass der Blutdruck im Gefolge weiterer Injectionen nicht mehr genügend hoch und auf nicht genug lange angestiegen ist, um einen nach der beschriebenen Methode noch zil beobachtenden $\mathrm{Zu}$ wachs an Liquor klar zu erkennen zu geben. Es gibt aber auch Fälle, in denen der Blutrlruck auch nach zahlreichen Injectionen steigt und trotzdem kein Zuwachs an Liquor erfolgt. Offenbar ist der Apparat, der bei der Liquorbildung in Frage kommi, ebenso erschopflich wie alle anderen Vorrichtungen des lebenden Organismus. Unter diesen Bedingungen tritt auch, wie der Versuch IV lehrt, das plethysmographische Princip klarer zu Tage, das Steigen des Liquors in der Röhre wird dann vorwiegend durch dessen Verdrängung von dem sich vergrössernden Gehirne bewirkt.

Ich wende mich nun zu der Frage, ob die hier erschlossene Neubildung von Liquor als das Vorbild eines normalen Vorganges

1) Pflüger's Archiv Bd. 76. 
angesehen werden kann. Es sind dies in erster Reihe die hohen Blutdruckswerthe, welche Zweifel nach dieser Richtung hin erwecken könnten. Wir wissen ja bis hente nicht, ob eine Blutdruckshöhe von $300 \mathrm{~mm} \mathrm{Hg}$ sich jemals im Laufe des Lebens einstellen kann.

Trotz dieser Bedenken ist es berechtigt, in den geschilderten Vorgängen ein Vorbild der physiologischen und wahrscheinlich auch der pathologischen Liquorbildung, allerdings in einer durch Uebertreibung modificirten Weise, zu erblicken. Denn einerseits lehrt der Versuch V, dass in Folge der Dyspnoë das Liquormaximum ein ähnliches Verhalten zeigt, wie jenes nach den Extractinjectionen. Andererseits habe ich auch Versuche ausgeführt, in denen der Blutdruck dureh Anwendung schwächerer Extractdosen nicht bis zu jener abnormen Höhe getrieben worden ist, wie in den zuvor angeführten Experimenten. Es war demgemäss auch jeder einzelne Liquorzuwachs geringer und manchmal so wenig imponirend, dass ich denselben, wenn ich nicht die Wirkung von starken Injectionen gekannt hätte, vielleicht als etwas Zufälliges, Unwesentliches beurtheilt hätte.

In einem so übertriebenen Maasse, wie es oben an der Hand von Versuchen geschildert worden ist, dürfte der Liquor in der Norm wohl kaum erzeugt werden, andererseits kann aber die Vorstellung der älteren Forscher auf diesem Gebiete, welche den Liquor als "Vapor" und seine Bildung als "Aushauchen" bezeichnet haben, - sit venia verbo - als untertrieben angesehen werden.

Um ein vollständiges Bild von dem Mitgetheilten zu gewinnen, erscheint es geboten, noch einen Punkt zur Sprache zu bringen.

Von dem Standpunkte ausgehend, dass das hyperämische Gehirn den Liquor transsudirt, muss man die Frage aufwerfen, wie findet denn der Zuwachs an Flüssigkeit in den durch das geschwellte Gehirn verengten Liquorräumen Platz?

Falkenheim und Nauny n behaupten, „dass die Resorptionsgrösse des Liquors von der Höhe des arteriellen Druckes ganz und gar unabhängig ist". Wie sollte nun bei einer intensiven Gehirnhyperämie in Folge hohen Blutdruckes der neugebildete Liquor in den durch die Gehirnschwellung verengten Liquorräumen Raum finden? Solange die Messcanüle da ist, kann der zuwachsende Liquor sich in diese entleeren; aber wenn dieser Raum dem Liquor nicht zur Verfügung steht?

Ich balte indessen die Frage nach der Finwirkung des Blut- 
druckes auf die Resorptionsgrösse des Liquors für nicht gelöst. Die Beobachtungen Falkenheim's und Naunyn's sind gewiss zutreffend, aber sie beziehen sich doch nur auf die von ihnen gewählten Versuchsbedingungen. Die genannten Forscher liessen eine physiologische Kochsalzlösung unter einem bestimmten Drucke in den Rückgratscanal einfliessen und hemerkten, dass, wenn durch Ligatur der Aorta thoracica oder durch Strychnininjection der Blutdruck gestiegen war, der Einfluss jener Lösung gehemmt wurde. So lehrreich diese Versuche nach mancher Richtung hin sind, auf die hier diseutirte Frage können dieselben keine Anwendung finden, da es in Bezug auf die letztere vor Allem darauf ankommt, zu erfahren, nicht, wie sich der Einfluss, sondern wie sich der Ausfiuss nach Einführung des hohen Blutdruckes verhält. Nur die Beobachtung des letzteren könnte uns einen Einblick in das Verbältniss zwischen der Höhe des Blutdruckes und der Resorptionsgrösse des Liquors gewähren. Ich selbst habe hierüber schon einige Erfahrungen gesammelt. Da dieselben aber noch nicht publicationsfähig sind, muss ich mich hier nur mit dem Hinweise begnügen, dass die Supposition, das durch seinen wachsenden Blutgehalt schwellende Gehirn könnte dem neugebildeten Liquor durch Verdrängung des früher gebildeten Raum schaffen, mit den von Falkenheim und Naunyn errungenen Erfahrungen nicht collidirt.

Ich glaube darum eine Resorption auch bei den oben mitgetheilten Versuchen $(\mathrm{I}-\mathrm{V})$ nicht ausschliessen zu können, und dies schon aus dem Grunde, weil ich den Liquor in der Röhre aufsteigen liess. Denn aus den Untersuchungen von Naunyn und Schreiber ${ }^{1}$ ) einerseits und jenen von Falkenheim und $\mathrm{Naunyn}{ }^{2}$ ) andererseits folgt, dass die Resorption des Liquor von dem auf denselben lastenden Drucke abhängt. Es könnte somit die Liquorsäule, zumal wenn dieselbe nach wiederholten Extractinjectionen hoch angestiegen ist, immerhin in der Phase des fallenden Blutdruckes einen Druck auf die in den Liquorräumen sich befindende Flüssigkeit ausgeübt baben.

$\mathrm{Ob}$ nun aber die von mir angefübrten Versuche von einer Liquorresorption thatsächlich begleitet waren oder nicht, ist für die Berechtigung der von mir aufgestellten Behauptung, dass der Liquor

1) Archiv für experimentelle Pathologie und Pharmakologie Bd. 14. 1881.

2) l. c. 
bei gesteigertem arteriellem Drucke neugebildet wird, ohne wesentlichen Belang, da ich meine Schlussfolgerungen auf das Anwachsen der Liquorsäure basirt habe. Ist thatsächlich in den Versuchen (I-IV) Liquor resorbirt worden, dann würde nur so viel daraus zu folgern sein, dass die Liquorsäulen um die resorbirte Menge kleiner ausgefallen sind, dass demgemäss die Neubildung der Cerebrospinalflüssigkeit böher anzuschlagen sei. Den oben mitgetheilten Zahlen kommt demgemäss schon aus diesem Grunde nur ein relativer Werth zu.

Es wurde oben bemerkt, dass die Zunahme der Liquorsäule auch auf einer Hemmung der Resorption beruhen könnte. Würde man, um von einer bestimmten Vorstellung auszugehen, etwa die Annahme machen, dass die Ahflusswege contractil sind und durch die Extractinjection zur Contraction gebracht werden, so wären die bei den Versuchen I-IV gesammelten Frfahrungen noch nicht erklärt, da durch jede Zusammenziehung zwar der Liquor vermebrt, aber diese Vermehrung durch Erschlaffung der Ausflussbahnen wieder wettgemacht werden würde. Um eine Erklärung herbeizuführen, müsste man an die eben angeführte Hypothese noch eine andere Hilfshypothese anreihen, man müsste aunehmen, dass die Ausflusswege sich mit jeder neuen Injection stärker zusammenziehen, ohne sich in den Pausen zu dilatiren, damit das Liquormaximum mit jeder Injection wachsen könne. Man würde demnach, wenn man die gehemmte Liquorresorption zur Deutung der sub 1-6 angeführten Beobachtung heranziehen wollte, das Gebiet von unerwiesenen Speculationen betreten. Aber nicht genug an dem, man würde sich mit der durch directe Beobachtung sichergestellten Thatsache, dass der Liquor während des hohen Blutdruckes aus dem Gehirne hervorperlt, also neugebildet wird, in Widerspruch setzen. Es entspricht somit nur den Gesetzen der Logik, wenn wir den eben erwähnten Versuch einer Deutung als unberechtigt bei Seite lassen.

Fin Rückblick auf das Mitgetheilte lehrt demnach, dass bei hohem Blutdrucke und gleichzeitiger Hyperämie des Gehirns eine Neubildung des Liquors erfolgt und dass dieselbe durch zwei differente Methoden, durch directe Beobachtung des aus dem Gehime hervorsickernden Liquors und durch volumometrische Untersuchungen erwiesen werden kann. 